\title{
Temperature Assimilation into a Coastal Ocean-Biogeochemical Model: Assessment of Weakly and Strongly-Coupled Data Assimilation
}

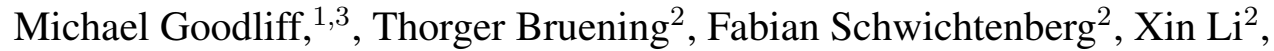 \\ Anja Lindenthal ${ }^{2}$, Ina Lorkowski ${ }^{2}$, Lars Nerger, ${ }^{1 *}$ \\ ${ }^{1}$ Alfred-Wegener-Institut Helmholtz-Zentrum für Polar- und Meeresforschung, Bremerhaven, Germany \\ ${ }^{2}$ Bundesamt für Seeschifffahrt und Hydrographie, Hamburg, Germany \\ ${ }^{3}$ now at Cooperative Institute for Research in the Atmosphere, Colorado State University, Fort Collins, USA \\ *Corresponding author; phone: +49(471)4831-1558; E-mail: lars.nerger@awi.de
}

\begin{abstract}
Satellite data of both physical properties as well as ocean colour can be assimilated into coupled ocean-biogeochemical models with the aim to improve the model state. The physical observations like sea surface temperature usually have smaller errors than ocean colour, but it is unclear how far they can also constrain the biogeochemical model variables. Here, the effect of assimilating satellite sea surface temperature into the coastal ocean-biogeochemical model HBM-ERGOM with nested model grids in the North and Baltic Seas is investigated. Weakly and strongly-coupled assimilation is performed with an ensemble Kalman filter. For weaklycoupled assimilation, the assimilation only directly influences the physical variables, while the biogeochemical variables react only dynamically during the 12-hour forecast phases in between the assimilation times. For strongly-coupled assimilation, both the physical and biogeochemical variables are directly updated by the assimilation. The strongly-coupled assimilation is assessed in two variants using the actual concentrations and the common approach to use the logarithm of the concentrations of the biogeochemical fields. In this coastal domain, both the weakly and strongly-coupled assimilation are stable, but only if the actual concentrations are used for the strongly-coupled case. Compared to the weakly-coupled assimilation, the strongly-coupled assimilation leads to stronger changes of the biogeochemical model fields. Validating the resulting field estimates with independent in situ data shows only a clear improvement for the temperature and for oxygen concentrations, while no clear improvement of other biogeochemical fields was found. The oxygen concentrations were more strongly improved with strongly-coupled than weakly-coupled assimilation. The experiments further indicate that for the strongly-coupled assimilation of physical observations the biogeochemical fields should be used with their actual concentrations rather than the logarithmic concentrations.
\end{abstract}

Keywords

Data Assimilation; biogeochemistry; North Sea; Baltic Sea 


\section{Introduction}

In recent years, ocean forecasting has become more common, e.g. with the European Copernicus Marine Environment Monitoring Service (CMEMS). In Germany, the Federal Maritime and Hydrographic Agency (BSH) operates a forecasting system for the North and Baltic Seas based on the HIROMB-BOOS model (HBM, see, e.g., Bruening et al., 2014). The national monitoring duties, e.g. to fulfil the European Marine Strategy Framework Directive (MSFD) require monitoring the seas with regard to water quality and hence also for the ecosystem. Given that in situ observations are sparse and hence insufficient for the monitoring, the extension of forecast models with an ecosystem component is required. A coupled ocean-biogeochemical model, which simulates phytoplankton and nutrients, can represent e.g. eutrophication, but can potentially also predict harmful algal blooms.

To initialise model forecasts, different observations can be assimilated. Satellite observations, e.g. of temperature or sea level, are frequently available measurements of the sea surface. The assimilation of physical observations to constrain the physical ocean model is common practice. However, it has been found that the assimilation of these observations to constrain the physical ocean state can deteriorate the biogeochemical (BGC) fields. For the North Atlantic, Berline et al. (2007) found that the assimilation of sea surface temperature (SST) and sea surface height (SSH) data changed the mixed layer so that much higher vertical nutrient fluxes appeared in the mid-latitudes and sub-tropics, which caused deteriorated phytoplankton concentrations. Also, While et al. (2010) reported increased nutrients and in consequence overestimated primary production and chlorophyll concentrations in the subtropical gyres and at the equator. Similar increased upward flux of nutrients and corresponding increased production was found by Raghukumar et al. (2015) in the California Current System. To correct for spurious changes by the data assimilation, corrections to the nutrient fields have been proposed (While et al., 2010; Shulman et al., 2013) while Park et al. (2018) suggests to reduce the assimilation effect around the Equator.

There are also observations of the ocean colour, from which e.g. concentrations of chlorophyll or diffuse attenuation rates are derived. In particular, chlorophyll concentrations have been used to directly influence the 
BGC model state (e.g. Nerger and Gregg, 2007, 2008; Gregg, 2008; Ciavatta et al., 2011; Ford et al., 2012; Ford and Barciela, 2017). However, the data errors are higher for chlorophyll than for physical quantities like SST. Further, satellite chlorophyll observations have particularly high uncertainties in coastal waters, because the standard processing, like the ocean-colour algorithm by Hu et al. (2012) commonly used in the processing of MODIS data, is only valid for clear case-1 waters and the availability of data sets processed for the coastal regions is very limited. Another data source on BGC quantities are in situ data, e.g. of nitrate. While these data are also available below the surface, they are much more sparse than satellite data, which strongly limits their applicability for data assimilation.

In coupled data assimilation, one can classify the data assimilation approach depending on which model fields are influenced by which data type. The studies mentioned above performed so-called 'weakly-coupled' assimilation, by assimilating observations of the ocean physics into the physical model component or assimilating observations of BGC variables into the ecosystem component of the coupled model. A more sophisticated approach is the 'strongly-coupled' data assimilation. In this case, one uses cross-covariances between the physical and BGC model components to let the assimilation algorithm utilise physical observations to directly update also BGC model variables. Strongly-coupled data assimilation is challenging because it depends on the quality of the estimated cross-covariances and requires that compatible assimilation methods are used in the different model components. This appears to be a particular issue for the assimilation into coupled atmosphere-ocean models as the recent review by Penny et al. (2017) shows.

Only a limited number of studies have so far considered the combined assimilation of physical and BGC observations. However, while assimilating both physical and BGC observations, the published studies (Anderson et al., 2000; Ourmières et al., 2009; Song et al., 2016b,c; Mattern et al., 2017) all set the cross-covariances between different variables to zero. Thus, in terminology of coupled data assimilation, only weakly-coupled data assimilation was performed, in which the direct assimilation influence of the physical observation was only on the physical model fields, while the BGC observations had only a direct influence on the modelled BGC concentrations. Only 
during the subsequent model forecast, or in iterations of a variational minimisation method, the changed model fields interacted. Nonetheless, the studies find that the combined weakly-coupled assimilation of physical and BGC observations improved the overall consistency of the coupled model state.

Until now, strongly-coupled assimilation into a coupled ocean-BGC model was only studied by Yu et al. (2018). The study used an idealised configuration of a channel with wind-induced upwelling and synthetically generated observations, i.e. a twin experiment. Different combinations of weakly and strongly-coupled assimilation assimilating either physical (SSH, SST and temperature profiles) or BGC data (surface chlorophyll and nitrogen profiles) or assimilating both data types were conducted. The experiments showed that in this idealised case, the cross-covariances between the physical and BGC model variables contain useful information that can be used in the strongly-coupled assimilation.

In this study, the effect of strongly-coupled assimilation in a realistic ocean-BGC model is assessed. For this purpose, the data assimilation is performed on the coastal coupled ocean-BGC model HBM-ERGOM configured for the North and Baltic Seas using two nested meshes. An earlier model version of the physical circulation model (BSHcmod, Dick et al., 2001; Kleine, 2003) with a simpler model configuration without nesting was used in previous studies (Losa et al., 2012, 2014; Nerger et al., 2016) to assess the influence of SST assimilation. Only satellite SST data is assimilated here and the effect of both weakly and strongly-coupled assimilation is assessed. A particular focus is on the question whether the strongly-coupled assimilation of SST data, i.e. direct joint update of both the physical and BGC model fields, improves the model state in this coastal setup.

A further aspect examined here is the different effect when treating the BGC model fields in the assimilation using the actual concentrations or the logarithm of them. Based on the fact that the chlorophyll concentrations can be well described as log-normally distributed (Campbell, 1995), many studies employing ensemble Kalman filters (e.g. Nerger and Gregg, 2007, 2008; Ciavatta et al., 2011; Pradhan et al., 2019) or optimal interpolation (Ford et al., 2012) have applied the data assimilation to the logarithm of the concentrations or by applying a socalled anamorphosis transformation (Doron et al., 2011). For the BGC assimilation with variational methods, 
Song et al. (2016a) have developed a method to treat lognormal concentration distributions. On the other hand, the actual concentrations have been used by other studies applying ensemble Kalman filters (e.g. Carmillet et al., 2001; Natvik and Evensen, 2003; Mattern et al., 2010; Yu et al., 2018) and 3-dimensional variational assimilation (Teruzzi et al., 2014). The latter study also discusses that actual concentrations were used because only then the typical structure of vertical chlorophyll profiles was preserved. In this study, both cases of actual and logarithmic concentrations are examined.

This study is structured as follows: Section 2 describes the coupled model HBM-ERGOM. The data assimilation methodology and the observations assimilated and used for validation are described in Sec. 3 while Sec. 4 describes the setup of the data assimilation experiments. The assimilation effect is assessed in Sec. 5 for using actual biogeochemical concentrations and in Sec. 6 for the case of the logarithmic treatment of the biogeochemical variables. The results are discussed in Sec. 7 while conclusions are drawn in Sec. 8.

\section{HBM-ERGOM model}

The model used here is the HIROMB-BOOS-Model (HBM) coupled to the BGC model ERGOM. HBM is currently used operationally, without data assimilation, by the $\mathrm{BSH}$ in a similar configuration as used here. The coupled HBM-ERGOM configuration is currently used pre-operationally at the BSH.

$\mathrm{HBM}$ is a three-dimensional hydrostatic circulation model using the primitive equations. It uses spherical horizontal and generalised vertical coordinates (Kleine, 2003). The model domain extends from $4^{\circ} \mathrm{W}$ to $30.5^{\circ} \mathrm{E}$ and from $48.5^{\circ} \mathrm{N}$ to $60.5^{\circ} \mathrm{N}$ in the North Sea and to $66^{\circ} \mathrm{N}$ in the Baltic Sea. A nested configuration of the model is used with two domains shown in Fig. 1. The coarser grid covers the entire North Sea and Baltic Sea. It has horizontal grid spacing of about $5 \mathrm{~km}\left(5^{\prime}\right.$ in longitude and $3^{\prime}$ in latitude) and 36 vertical layers. In the region of German territorial waters in the North Sea and Baltic Sea, a finer grid with a horizontal resolution of about $900 \mathrm{~m}$ (50" in longitude and 30" in latitude) and 25 vertical layers is nested into the coarse grid using a 2-way nesting. 
In the North Sea, the model configuration has a northern open boundary in the coarse mesh, which is closed with a sponge layer. Within this layer, the temperature and salinity are restored towards monthly mean climatological values (Janssen et al., 1999). A similar sponge region is included at the entrance to the English Channel. A two-dimensional model for the North East Atlantic, which is run separately by the BSH, provides information on external surges at the open boundaries. Tidal forcing is implemented using 14 tidal constituents and flooding and drying of tidal flats is applied (Bruening et al., 2014). The atmospheric forcing at the surface is based on meteorological forecast data provided by the German Weather Service (DWD). River runoff is prescribed as freshwater fluxes at the boundaries opened in the regions of main rivers. Further, HBM includes a sea-ice model component that describes sea ice thermodynamics and incorporates Hibler-type dynamics (Hibler, 1979).

The BGC model ERGOM was originally developed by Neumann (2000) for the Baltic Sea and upgraded later by Maar et al. (2011) for the ecosystems in the North and Baltic Seas. ERGOM simulates the BGC cycling in the coastal seas using three phytoplankton groups (Cyanobacteria, Flagellates, Diatoms), two zooplankton size groups, four nutrient groups (nitrate, ammonium, phosphate, and silicate), two detritus groups (N-Detritus and Si-Detritus), oxygen and labile dissolved organic nitrogen in the water column (IDON, Neumann et al., 2015). The phytoplankton and zooplankton groups are expressed in nitrogen concentrations. The chlorophyll-a concentration and the Secchi depth are computed diagnostically (Doron et al., 2013; Neumann et al., 2015). Riverine load inflow of nutrients was derived from climatological data for major rivers. The boundary conditions for the BGC state variables are from the World Ocean Atlas (WOA05) as described by Maar et al. (2011). ERGOM is coupled one-way to HBM so that the physical fields influence the biogeochemistry, which itself does not influence the physics.

\section{Data Assimilation}

The data assimilation is performed using the ensemble-based Error-Subspace Transform Kalman filter (ESTKF Nerger et al., 2012b) provided by the Parallel Data Assimilation Framework (PDAF, Nerger et al. (2005); Nerger 
and Hiller (2013)), which are described in this section.

\subsection{Parallel Data Assimilation Framework}

The Parallel Data Assimilation Framework (PDAF, Nerger et al. (2005); Nerger and Hiller (2013), http://pdaf.awi.de) is an open-source software environment for ensemble data assimilation. It simplifies the implementation of the data assimilation system with existing numerical models by providing support to modify the model to compute ensemble forecasts and by providing fully implemented ensemble data assimilation methods. For the data assimilation, the model code is augmented by subroutine calls to PDAF. This changes the parallelisation of the model, so that it can simulate an ensemble of model states, which are then used in the analysis step of the data assimilation, where the observational information are incorporated into the model.

\subsection{Error-Subspace Transform Kalman Filter}

The data assimilation method used here is the Error-Subspace Transform Kalman Filter (ESTKF, Nerger et al., 2012a). The ESTKF is an efficient variant of the ensemble Kalman filter, which uses an ensemble of $N_{e}$ model states to represent the state estimate, as the ensemble mean, and its uncertainty by the ensemble spread. For an overview of different filter methods, see Vetra-Carvalho et al. (2018).

The ESTKF performs a sequential assimilation by alternating forecast phases and analysis steps. In the forecast phase, all model states in the ensemble are integrated by the model until the time when observations become available. Then, the analysis step is computed in which the observational information is assimilated into the model states.

Compared to the classical ensemble Kalman filter (EnKF Evensen, 1994; Burgers et al., 1998), the analysis step of the ESTKF is a particularly efficient formulation because it takes into account that the number of the degrees of freedom for the analysis update is given by $N_{e}-1$, while the EnKF computes the update according to the usually much higher number of observations (see Nerger et al. (2005) for a comparison of the EnKF with 
the SEIK filter, which has the same efficiency as the ESTKF). Mathematically, the ensemble describes the degrees of freedom by spanning an error-subspace of dimension $N_{e}-1$, which motivates the name of the filter method. In the analysis step, the ESTKF uses ensemble-sampled error covariances of the model forecast, the observation error, and the observational values to estimate the true state of the system. The ESTKF does this as follows by computing transformation weights. Let $\mathbf{X}_{k}$ denote an ensemble matrix at time $k$ in which each of the $N_{e}$ columns represents one model state. The transformation of the forecast ensemble, $\mathbf{X}_{k}^{f}$ into the analysis ensemble, $\mathbf{X}_{k}^{a}$ is given by

$$
\mathbf{X}_{k}^{a}=\overline{\mathbf{X}}_{k}^{f}+\mathbf{X}_{k}^{f} \mathbf{W}_{k}
$$

where the overbar denotes the ensemble mean and $\mathbf{W}_{k}$ is a transformation matrix of size $N_{e} \times N_{e}$. Given that the degrees of freedom given by the ensemble are $N_{e}-1$, this transformation matrix is calculated in an error-subspace of dimension $N_{e}-1$ at time $k$. Below, we omit the time index $k$, as all calculations of the analysis step are at this time. The transformation matrix is computed as follows. First, the ensemble states are projected onto the error subspace by

$$
\mathbf{L}=\mathbf{X}^{f} \mathbf{T},
$$

where $\mathbf{T}$ is a projection matrix of size $N_{e} \times\left(N_{e}-1\right)$ given by the set of equations

$$
\mathbf{T}_{j, i}= \begin{cases}1-\frac{1}{N_{e}} \frac{1}{\sqrt{\sqrt{N_{e}}}+1}, & \text { for } i=j, j<N_{e} \\ -\frac{1}{N_{e}} \frac{1}{\sqrt{\sqrt{N_{e}}}+1}, & \text { for } i \neq j, j<N_{e} \\ -\frac{1}{\sqrt{N_{e}}}, & \text { for } j=N_{e} .\end{cases}
$$

Now the matrix

$$
\mathbf{A}^{-1}=\rho\left(N_{e}-1\right) \mathbf{I}+\left(\mathbf{H} \mathbf{X}^{f} \mathbf{T}\right)^{T} \mathbf{R}^{-1}\left(\mathbf{H} \mathbf{X}^{f} \mathbf{T}\right)
$$

of size $\left(N_{e}-1\right) \times\left(N_{e}-1\right)$ is computed. Here, $\rho$ is the so-called forgetting factor, which is chosen as $0 \leq \rho \leq 1$ and inflates the ensemble variance to stabilise the filter process. $\mathbf{I}$ is the identity matrix and $\mathbf{H}$ is the observation operator which computes the model equivalent to the observations so that one can write $\mathbf{y}=\mathbf{H} \mathbf{x}^{f}+\eta$ where $\mathbf{y}$ is the observation vector of size $N_{y}, \mathbf{x}^{f}$ is a forecast state vector and $\eta$ is the observation error, which is assumed to be Gaussian with observation error covariance matrix $\mathbf{R}$. 
The weight matrix $\mathbf{W}$ in Eq. (1) is now computed as the sum of two terms

$$
\mathbf{W}=\overline{\mathbf{W}}+\tilde{\mathbf{W}} .
$$

Here, $\overline{\mathbf{W}}$ contains in each column the vector

$$
\overline{\mathbf{w}}=\mathbf{T A}\left(\mathbf{H} \mathbf{X}^{f} \mathbf{T}\right)^{T} \mathbf{R}^{-1}\left(\mathbf{y}-\mathbf{H} \overline{\mathbf{x}}^{f}\right)
$$

which performs the transformation of the ensemble mean, while the ensemble perturbations are transformed by

$$
\tilde{\mathbf{W}}=\sqrt{N_{e}-1} \mathbf{T} \mathbf{A}^{1 / 2} \mathbf{T}^{T} .
$$

Here $\mathbf{A}^{1 / 2}=\mathbf{U S}^{1 / 2} \mathbf{U}^{T}$ is the symmetric square root of $\mathbf{A}$ computed from the eigenvalue decomposition $\mathbf{A}=$ $\mathrm{USU}^{T}$

The degrees of freedom provided by the ensemble are too small to successfully assimilate the large number of satellite observations. Due to this, the ESKTF is applied here with a localised analysis as for the LSEIK filter (Nerger et al., 2006). Namely, the model state of each vertical column of the model grid is updated separately taking only observations into account that lie within a specified influence radius around the water column. Further, the observations are weighted according to their distance to reduce the influence of remote observations and to generate a smooth analysis field. For the weighting, the inverse observation error covariance matrix in Eq. (4) is multiplied element-by-element with a diagonal matrix constructed using the regulated localization of Nerger et al. (2012a) with a correlation function given by the fifth-order polynomial of Gaspari and Cohn (1999). This function mimics a Gaussian function and varies between one at zero distance and zero at the distance of the influence radius.

Since the model uses nested grids with different resolutions, one has to adapt the localisation. Here, the influence radius is chosen according to the location of the observation, as is depicted in Fig. 2. Thus, an observation located in the coarse grid is only taken into account for model grid points within the radius $r_{\mathrm{g}}$, while an observation located in the fine grid is only taking into account within the radius $r_{\mathrm{f}}$. Accordingly, the analysis update of a water column on the coarse grid also takes into account observations on the fine grid (vice versa for the update on the 
fine grid) if the grid point is sufficiently close to the fine grid. This ensures a smooth transition of the analysis field across the boundary of both grids.

\subsection{Observations}

In the experiments, satellite observations of the sea surface temperature are assimilated. These are measured with the Advanced Very High Resolution Radiometer (AVHRR) aboard polar orbiting NOAA satellites and processed by the BSH. Composites over 12 hours are used which are interpolated onto the two nested model grids. The composites use the satellite information over the 12-hour time window before the analysis step. Given that the radiometer provides only data for clear-sky conditions, the data coverage can vary significantly as shown in Fig. 3. This is particularly noticeable in the rather small fine grid region for the German coastal regions, where even 12-hour time windows with zero coverage can exist.

For the validation of the assimilation results, a data set of in situ data is used. The data set includes data from the International Council for the Exploration of the Sea (ICES Dataset on Ocean Hydrography. The International Council for the Exploration of the Sea, Copenhagen. 2016) and the German Oceanographic Data Center (DOD, http://seadata.bsh.de/csr/retrieve/dod_index.html) operated by the BSH. Apart from water temperature and salinity, the data set also includes measured concentrations of oxygen, nitrate, ammonium, phosphate, silicate, and chlorophyll, which can be used to assess the corresponding concentrations in the ERGOM model. The validation of the assimilation experiments will focus on the surface and will be conducted for both the fine and coarse model grids.

\section{Experimental Setup}

The assimilation experiments are conducted over the time period from April to July 2012 with an analysis update after each $12 \mathrm{~h}$. An ensemble of 40 model states is used. The initial physical ocean state (i.e. ensemble mean) is provided by the operational run of the HBM model at the BSH. The BGC model state was initialised on 1st November 2011 using for the Baltic Sea an initial state provided by the Danish Technical University (generated 
by the model of Maar et al. (2011) by M. Maar, personal communication) and for the North Sea an initial state generated by the model of Lorkowski et al. (2012). The ensemble perturbations were computed using 2nd-order exact sampling (Pham et al., 1998) using the variability of the model state in a forecast run of the HBM-ERGOM model for April 2012.

The state vector for the assimilation jointly includes the model fields on both nested model grids (similar to Barth et al., 2007) and consists of physical and BGC parts on both nested model grids. For the physical part the state vector includes the SSH and the 3-dimensional temperature, salinity, and horizontal velocities. For ERGOM, all 13 prognostic pelagic and 2 benthic variables as well as the Secchi depth and chlorophyll concentration are included in the state vector. The two latter diagnostic variables are, however, only included to access their ensemble values, but they are not directly updated by the analysis step of the LESTKF. For the localisation of the analysis step an influence radius for the observations of $50 \mathrm{~km}$ is used for the coarse grid, while $9 \mathrm{~km}$ are used for the fine grid. An inflation of the ensemble variance with a forgetting factor of $\rho=0.95$ is used. For the assimilation of the SST observations, an observation error standard deviation of $0.8^{\circ} \mathrm{C}$ is assumed as in Losa et al. (2014) for both model grids.

Two assimilation experiments are performed to assess the different effects of the weakly and strongly-coupled assimilation. The experiment WEAK assimilates the SST observations so that only the physical model fields in the state vector are directly updated. The BGC model fields react only dynamically to the changed physical conditions during the next forecast phase of 12 hours. In contrast, in the experiment STRONG both the physical as well as BGC model fields are directly updated. Thus, the strongly-coupled assimilation uses the multivariate ensembleestimated cross-covariances between the SST and the BGC variables to compute an update of the biogeochemistry. Further, the experiment FREE was performed in which the ensemble was integrated without assimilating observations.

The experiment STRONG is performed in two variants. STRONG-lin performs the assimilation using the actual concentrations of the BGC variables. In this case, the statistical update computed by the LESTKF can 
result in negative concentrations. As in Yu et al. (2018), these values were reset to zero, but occurred only in a few cases in the experiments. The experiment STRONG-log performs the assimilation using the logarithm of the concentrations

The experiments allow us to assess whether the cross-covariances between the SST and the BGC model fields are sufficiently well estimated to result in an improvement of the BGC fields. For this, the root mean square error (RMSE) and the mean error (bias) between the state estimate from each data assimilation experiment with regard to the in situ validation data are computed. To assess the impact of the SST data on the modelled surface temperature and salinity we also compute the RMSE with regard to the assimilated data as well as RMSE and bias with regard to independent in situ data of temperature and salinity.

\section{Results}

To analyse the assimilation results, first the influence on the surface temperature and salinity are assessed. Then, the effect of the weakly-coupled assimilation on the biogeochemical model fields is examined, and finally, the effect of the strongly-coupled assimilation is assessed.

\subsection{Influence of the assimilation on surface temperature and salinity}

The effect of assimilating satellite SST data on the physical ocean state was already discussed by Losa et al. (2012) and Losa et al. (2014), so no detailed analysis is performed here. Figure 4 shows the RMSE with regard to the assimilated SST observations for the analysis and forecast fields each 12 hours as a time series for both model grids. For the forecasts, the RMSE is computed with observations that have not yet been assimilated. Given that the coverage of the SST observations varies in between the analysis times, the observations at the forecast time are partly independent, while they are not independent for the analysis. Nonetheless, the values of the RMSE for the forecast and analysis are very similar. Since HBM-ERGOM uses a one-way coupling between the physical and biogeochemical models, the physical model fields are identical in the experiments WEAK and STRONG. The 
assimilation of SST data pulls the SST in the model toward the observations while accounting for the uncertainty in both the model state and the observations. Further, through the covariances estimated by the ensemble, the observational information is interpolated spatially and unobserved model fields are modified. For the coarse grid (upper panel) the RMSE of the forecast and analysis is clearly reduced compared to the free run. For the fine grid (lower panel), the RMSE is also reduced, but the fluctuations of the errors between the different analysis times are larger and the overall error-reduction is smaller. Namely, the average RMSE is reduced in the forecast by $0.21^{\circ} \mathrm{C}$ (from $1.02^{\circ} \mathrm{C}$ for the free run to $0.81^{\circ} \mathrm{C}$ ) on the coarse grid while the reduction is $0.14^{\circ} \mathrm{C}$ (from $0.89^{\circ} \mathrm{C}$ to $0.75^{\circ} \mathrm{C}$ ) on the fine grid. Nonetheless, on the fine grid the error is lower on average compared to the coarse grid. The strong variations of the RMSE, which are particularly visible for the fine model grid, are mainly due to the varying data coverage in between the analysis times. Both the number of observations and the observation locations varied strongly, so that the computation of the RMSE covers different regions and a strongly varying number of comparison points, which leads to sampling errors. For example, on May 10th at $12 \mathrm{~h}$, when the highest RMSE occurs on the fine grid, only 893 grid points out of 124000 overall surface grid points were observed. Just before, at $0 \mathrm{~h}$ on May 10th, there were 12275 observed grid points and at 0h on May 11th, 2464 observations were available. Likewise on May 11 at $0 \mathrm{~h}$ there is a very low number of only about 2000 observed grid points in the coarse grid and a particularly small RMSE. Apart from this effect, the data assimilation process of alternating analyses and forecasts induces a gradual modification of the ocean state over time as is visible from the small difference between the RMSE in the forecasts and analyses, but larger RMSE in the free run. Accordingly, the RMSE of the forecast or analysis at a certain time, depends on the observations that have been assimilated before. Overall, the variability of the RMSE is mainly caused by the coverage of the observations and less by specific oceanographic events.

While the spatially averaged RMSE of the forecasts shows only small reductions by the data assimilation up to $0.21^{\circ} \mathrm{C}$ (and $0.24^{\circ} \mathrm{C}$ for the analysis states), the assimilation influence is locally much larger. Fig. 5 shows the effect of the assimilation as an average over July 2012. The RMSE in the FREE run (upper row) is mainly below $0.8^{\circ} \mathrm{C}$ in both grids, but it is larger in the western side of the English channel, in the region of the Norwegian 
trench, along the south-eastern coast of Sweden, the Gulf of Bothnia, and at the southern coast of Finland (see Fig. 1 for geographic information). Locally, the RMSE exceeds $4^{\circ} \mathrm{C}$. The data assimilation strongly reduces these high errors almost everywhere except in the far northern end of the Baltic Sea and in the English channel (middle row). In the fine grid, the error reductions are particularly visible at the southern coast of Sweden and along the German coast of the Baltic Sea. The bottom row of Fig. 5 shows the actual change in the temperature. In most regions of the model domain the assimilation has reduced the temperature. However, east of the islands Öland and Gotland, the temperature is increased up to $2^{\circ} \mathrm{C}$. Here, upwelling of cold water was present in the free model run, which is not present in the observations. The assimilation of the SST data increases the SST in the full water column hence decreasing the RMSE. Overall, the error reductions are similar to those described by Losa et al. (2012) and Losa et al. (2014) where SST data with a similar model was used without a refined nested grid. The comparison with the assimilated observations shows that the assimilation system is successful in incorporating the observational SST data.

Table 1 shows the RMSEs computed with regard to the in situ observations of SST over the full period from April to July 2012. The number of in situ data is overall low with 6674 points on the coarse grid and 800 points on the fine grid. On the coarse grid, the assimilation reduces the RMSE from $1.07^{\circ} \mathrm{C}$ in the FREE run to $0.92^{\circ} \mathrm{C}$ in the analysis. The forecast RMSE is only slightly larger with $0.925^{\circ} \mathrm{C}$. The RMSE of the FREE run is $1.15^{\circ} \mathrm{C}$ and hence larger than on the coarse grid. This is in contrast to the RMSE with regard to the assimilated satellite observations, where the RMSE on the fine grid is lower than on the coarse grid. The RMSE is reduced by the data assimilation to $1.05^{\circ} \mathrm{C}$. Overall the reduction of the RMSE is lower for the in situ data than the assimilated SST observations. The assimilation also reduces the warm bias of the model SST in both model grids. On the coarse grid, the bias is reduced by $62 \%$, while it is reduced by $58 \%$ on the fine grid. So, the reduction of the bias is overall larger than that of the RMSE.

The lower part of Table 1 shows the RMSE for surface salinity. Overall the changes to the salinity RMSE are very small. The changes are due to the direct update of the salinity field through the cross-covariances between 
the temperature and salinity, but also due to the fact that the assimilation also influences the velocities. The assimilation reduces the error on the coarse grid from 1.43 PSU to 1.39 PSU in the analysis. On the fine grid, the RMSE of the salinity is slightly increased by about $0.4 \%$ by the assimilation. While the changes in the RMSE and bias are statistically significant for the coarse grid only the change in bias is significant for the fine grid (at 95\% probability according to a paired t-test). Locally the largest changes happen in the transition zone between the salty North Sea (around 35 PSU) and the fresh Baltic Sea (5 to 8 PSU), i.e. the Danish Straits in the fine grid and the Skagerrak and Kattegat in the coarse grid. The assimilation also reduces the amount of bias by about $8 \%$. The model underestimates the salinity in the coarse grid, while it overestimates the salinity in the fine grid.

\subsection{Weakly-coupled assimilation effect on the biogeochemical model fields}

In the weakly-coupled data assimilation, only the physical model fields are directly updated by the LESTKF in the analysis step. The BGC model fields then react dynamically on the changed physical conditions during the following forecast phase. Table 2 shows the RMSE and bias computed with regard to the in situ data for 6 BGC variables. The changes are largest for oxygen with a reduction of the RMSE by $3.5 \%$ and bias by $17 \%$ on the coarse grid and a reduction of the bias by $64 \%$ on the fine grid. These changes are statistically significant at $95 \%$ probability using a paired t-test. Changes to other variables are generally smaller.

To get more insight into the changes to the biogeochemistry which are induced by the data assimilation, we examine the surface oxygen during the month of May 2012. Figure 6 shows monthly averaged oxygen concentration for the experiment FREE for both model grids. The in situ data values are plotted on top of the model fields. In the Baltic Sea, but also in the German Bight in the North Sea, the model mainly underestimates the oxygen concentration.

The bottom row of Fig. 6 shows the difference between the oxygen concentrations from the WEAK and FREE experiments averaged over May 2012. The dynamic reaction of the model on the assimilation is to increase the oxygen concentration by up to $18 \mathrm{mmol} / \mathrm{m}^{3}$ in the Baltic Sea, which reduces the model bias. The dynamic reaction 
on the assimilation is much smaller in the North Sea with increases and decreases up to $5 \mathrm{mmol} / \mathrm{m}^{3}$. Fig. 7 shows the comparison between the model concentrations and the in situ data as scatter plots. Consistent with Fig. 6, the main influence of the assimilation is to increase concentrations that are above $340 \mathrm{mmol} / \mathrm{m}^{3}$ in the experiment FREE. For the group of data points at about $350 \mathrm{mmol} / \mathrm{m}^{3}$ in the coarse grid this lead to a slight overestimation of oxygen. Since also larger concentrations that are generally too low in the model are further increased the overall assimilation effect is positive. Thus, the assimilation reduces the RMSE and the amount of bias with statistically significance (at 95\% probability). However, the correlation between the model and the situ data remains essentially unchanged. The overall assimilation effect is similar in April and June, while it is lower for July.

\subsection{Strongly-coupled assimilation effect on the biogeochemical model fields}

In the strongly-coupled data assimilation experiments STRONG-lin and STRONG-log, all BGC model fields are directly updated, together with the physical fields, by the LESTKF utilising the ensemble-estimated crosscovariances between the SST and the BGC fields. Thus, one expects a more directed and larger influence of the assimilation. If some BGC model field is not correlated with SST, the ensemble represents this relation (up to sampling error in the ensemble). In this section, the assimilation effect for the experiment STRONG-lin is examined, i.e. for the case that actual concentrations are used in the LESTKF. The experiment STRONG-log is discussed in Sec. 6 .

Table 3 shows the RMSE and bias with regard to the in situ data for the experiment STRONG-lin. The change in the RMSEs is slightly larger than for the weakly-coupled assimilation. The largest change happens for oxygen on the coarse grid where the RMSE is reduced by $4.7 \%$ in the experiment STRONG-lin, while it was only reduced by $3.5 \%$ in WEAK. Further, the amount of bias is now reduced by $24 \%$ compared to $17 \%$ in WEAK. On the fine grid the amount of bias is also more strongly decreased (by $89 \%$ ), while the RMSE is now increased by $1.9 \%$. The changes to the other fields are still small. Noticeable is a reduction of the bias for chlorophyll on both grids and for Silicate on the fine grid. The RMSE for chlorophyll was essentially unchanged in WEAK, but is increased slightly 
in STRONG-lin. Actually, in the eastern Gulf of Finland the chlorophyll concentration was unrealistically high during the first half of May in STRONG-lin. This effect will be further discussed in Sec. 7. Further, the biases for nitrate and phosphate are increased in STRONG-lin in the coarse grid, while they were marginally decreased in WEAK.

Figure 8 shows the change in the oxygen field averaged over May 2012. Compared to the weakly-coupled assimilation, the strongly-coupled assimilation results in larger changes up to $24 \mathrm{mmol} / \mathrm{m}^{3}$. Further the stronglycoupled assimilation leads to larger changes in the North Sea up to $10 \mathrm{mmol} / \mathrm{m}^{3}$. The bottom row of Fig. 7 shows the comparison between the model and in situ data for May 2012. The strongly-coupled assimilation further increases concentrations that were above $340 \mathrm{mmol} / \mathrm{m}^{3}$ in the experiment FREE compared to the experiment WEAK, which reduces both RMSE and bias on both grids for this month.

Several studies (e.g. Shulman et al., 2013; While et al., 2010; Yu et al., 2018) applied the assimilation of physical observations so that in the BGC model only nutrients are updated, instead of all BGC model fields. We performed an alternative experiment in which the phytoplankton, zooplankton, and detritus were excluded from the assimilation update. The assimilation influence on the RMSE and bias with regard to the in situ data is summarised in the right columns of table 3. With this update variant, the RMSE of nitrate, chlorophyll, oxygen, and silicate are reduced in both model grids by up to $2 \%$ compared to the case when all fields are updated. However, the amount of bias increased in particular for oxygen and chlorophyll concentrations with increases of $6 \%$ and $29 \%$, respectively. Note that here chlorophyll is particular because it is computed from the phytoplankton, which is not directly updated by the data assimilation in this experiments. In this experiment, the high concentrations in the Gulf of Finland were not present.

\section{Assimilation using logarithmic concentrations}

Above, the strongly-coupled assimilation was applied in the experiment STRONG-lin using the actual concentration values of the BGC fields in the state vector. As discussed in the introduction, chlorophyll concentrations can 
be well described as log-normally distributed (Campbell, 1995) which motivated many assimilation studies to use the logarithm of the concentrations in the state vector. The analysis step in the Kalman filter assumes normal error distributions for optimality and taking the logarithm of a log-normally distributed field results in a normal distribution. Likewise, this transformation is then applied to other BGC variables. While using actual concentrations appears to be statistically inconsistent with the assumptions of the Kalman filter, the studies using actual concentrations in the assimilation were also successful. This can be mainly explained by the fact that the assimilation using actual concentrations still results in corrections of the correct sign. However, the size of the correction will be different because normal distribution is symmetric while the log-normal distribution is skewed. Using the logarithm will typically lead to a tendency to more strongly increase concentrations. According to our experience, using the logarithm also leads overall to larger changes to the concentrations and a more sensitive assimilation system in particular for non-observed parts of the model fields like below the ocean surface. Due to this, Pradhan et al. (2019) introduced a vertical localisation to stabilise the assimilation update of subsurface variables. In this vertical localisation, the assimilation increment computed for the full water column is linearly reduced as a function of depth until it reaches zero at a prescribed depth (100m in Pradhan et al. (2019)).

In Sec. 5.3, we found that the strongly-coupled assimilation applied with the actual concentrations improved the oxygen concentrations but the changes to the other BGC fields were very small. Here, the strongly-coupled assimilation experiments of Sec. 5.3 are repeated using the logarithm of the BGC model fields (experiment STRONG$\log$ ) both with updating all fields of the BGC model and only updating the nutrients and oxygen. Using the logarithm of the concentrations in each ensemble state in the LESTKF, the cross-covariances used to update the BGC model fields are now computed from the logarithmic concentrations.

In the experiment STRONG-log, unrealistic concentrations developed already during the second half of April. The experiments were stopped at the end of May. Table 4 shows very high RMSEs for the case that the assimilation is performed over the full water column (The columns labelled with 'full vertical' in Tab. 4). The behaviour was different in the North Sea from the Baltic Sea. While in the Baltic Sea extreme RMSEs occur for all BGC fields, 
the RMSEs remain in a reasonable range for chlorophyll and silicate in the North Sea. Here mainly the northeastern region along the Norwegian Trench was affected by unrealistically high concentrations (not shown). When the phytoplankton variables were excluded from the DA update ('nutrients only' in Tab. 4) the RMSEs were lower. However, in the Baltic Sea the concentrations of most of the fields were still unrealistically high. In the North Sea silicate showed unrealistically high concentrations in the region of the Norwegian Trench while all other fields showed realistic concentrations. This is in contrast to the case when all fields are updated which resulted in realistic silicate concentrations.

When a vertical localisation is applied, the assimilation can be stabilised. With a localisation depth of $10 \mathrm{~m}$, the concentrations in the North Sea become realistic if all BGC fields are updated and the RMSEs are similar to those of the FREE experiment (Table 4, compare columns 2 and 5). However, for the Baltic Sea this localisation is not sufficient and even with a vertical localisation depth of $5 \mathrm{~m}$ the model fields show unrealistic concentrations. If only the nutrients are updated, only the nitrate concentrations in the Baltic Sea show unrealistic values in the Gulf of Finland and to a lesser extent in the southern Baltic Sea with vertical localisation. The unrealistic concentrations are not directly obvious from the value sof all RMSEs since the unrealistic concentrations can be very localised, e.g. in the eastern Gulf of Finland. Accordingly, they remain undetected if there is no in situ data available at this location. This case is exemplified for surface chlorophyll in Fig. 9. Here, the experiment WEAK (top left) results in concentrations of up to about $9 \mathrm{mg} / \mathrm{m}^{3}$ in the Baltic Sea. In the experiment STRONG-log without vertical localisation and update of all BGC fields (bottom left), high concentrations of chlorophyll appear in the Gulf of Bothnia and the Gulf of Finland. In particular, the isolated regions of high concentration at about $20^{\circ} \mathrm{E}, 62.5^{\circ} \mathrm{N}$ (with concentrations up to $100 \mathrm{mg} / \mathrm{m}^{3}$ ) and in the Gulf of Finland (with concentrations up to $22000 \mathrm{mg} / \mathrm{m}^{3}$ ) are unrealistic. The same holds for the isolated regions of near-zero concentration (e.g. at the western end of the Gulf of Finland). With a vertical localisation of 5m, the spurious high and low concentrations disappear everywhere except in the eastern Gulf of Finland, where still spuriously high concentrations exist. As there is no in situ data available at this location this issue is not detectable from the validation with the in situ data. In contrast, in the North Sea the chlorophyll field from WEAK and the two experiments STRONG-log updating all BGC variables 
with and without vertical localisation show only small differences and no unrealistic values.

\section{Discussion}

The assimilation of SST data into a coupled ocean-BGC model has two aspects: The effect on the physical state and the effect on the BGC model. For the physical component, the SST assimilation showed improvements of the SST when compared to independent in situ data. Changes to the salinity were small, but actually, no strong error correlation between SST and salinity is expected. This also holds for the velocity field, which was not further discussed above. While at a single analysis state the horizontal velocities were influenced, their overall change was small and the velocities in the North Sea are strongly influenced by tides. The assimilation also influences the model state below the surface. For example the strong temperature increases east of Öland and Gotland shown for the surface in Fig. 5 also occur in lower model layers. Thus, consistent with earlier studies (Losa et al., 2012, 2014; Liu and Fu, 2018) the full 3-dimensional physical model state was updated by the data assimilation and effects like the upwelling in July can be corrected. Nonetheless, the SST data cannot fully constrain the model and the assimilation of further observations like for sea surface salinity, sea surface height, velocities (like from HF radar observations, see e.g. Barth et al. (2010)) will be required. Further, the assimilation of subsurface in situ data will be required to further improve the lower layers for which surface data alone is not sufficient. For example in the Danish straits, dense water of high density can flow from the North Sea into the Baltic Sea close to the bottom, which will not be detected by surface observations (see Losa et al., 2012, 2014, for discussions on this issue).

For the effect on the BGC model state different cases exist. For the weakly-coupled case in which the BGC model fields react only dynamically to the changed physical state, the experiments show only small changes. In the validation with independent in situ data only the oxygen concentrations are changed to a statistically significant extent. This change in the oxygen concentration can be mainly attributed to the changed temperature that changed the solubility of oxygen. Actually, for July 2012 the change in oxygen concentrations has nearly the same pattern, but reversed sign, as the temperature change in the bottom row of Fig. 5. Other BGC variables did not show 
a clear improvement. Mainly, we expect that the processes in the ERGOM model would react to the changed temperature. Thus, the growth of the phytoplankton groups is modified which affects the nutrient concentrations. The assimilation did not directly modify the vertical velocity so that the vertical entrainment of e.g. nitrate is not modified. Anyway, this effect should only be present in the Baltic Sea and the Norwegian Trench, while the North Sea is shallow and usually well mixed. Given that the error in the BGC model state without data assimilation is rather large, and the dynamic reaction is small, the changes in the BGC state induced by the data assimilation are also small compared to its error.

The strongly-coupled assimilation resulted in larger changes of the BGC model fields. In particular oxygen was further improved. However, the dependence of oxygen solubility in temperature makes it well (anti-)correlated to temperature. This correlation is expected to be represented by the ensemble and hence the strongly-coupled assimilation should improve oxygen. The dependence of other BGC fields on temperature is not that direct. E.g. the nutrients will depend more strongly on the changed growth of the phytoplankton. Whether the ensembleestimated covariances can improve the model state also depends on the initial error in the BGC fields. Generally, the LESTKF, like any ensemble Kalman filter, perform a linear regression between the observed and unobserved model fields or locations (see e.g. Anderson, 2003). While the linear relationship will always hold for small errors (in the sense that a Taylor expansion could be truncated to the linear term), large errors will result in non-linear relationships. This is also expected for the nonlinear processes of a BGC model as was, e.g. discussed for the assimilation of satellite data on phytoplankton functional groups by Ciavatta et al. (2018). Perhaps, the errors in the BGC model state are here too large for the linear assumption. Overall, the corrections in our real-world application are smaller than those obtained in the idealized twin experiments performed by Yu et al. (2018).

The question whether BGC fields should be treated in the assimilation with their actual concentrations or with the logarithm of the concentrations is still open. In experiments using 3D variational assimilation, Teruzzi et al. (2014) found for chlorophyll that vertical covariances constructed using empirical orthogonal functions were less representative when logarithmic instead of actual concentrations were used. However, at least for chlorophyll the 
model of a log-normal concentration distribution was established (Campbell, 1995) and the dynamically generated ensemble used here should be able to represent the vertical covariances. For other variables than chlorophyll the distribution is less clear. The distribution of oxygen in Fig. 7 shows only a small range and does not appear to be log-normally distributed. Even more, the assimilation bases on the assumption that the error distribution is normal and the distribution of the errors does not need to follow the distribution of the field itself. Basing on this open discussion, the comparison of the experiments STRONG-lin and STRONG-log shows the different effects of applying the assimilation to the actual concentrations or to their logarithm. In particular, STRONG$\log$ leads to unrealistic concentrations. The positive influence of the vertical localisation shows that the linear regression of the surface temperature increments onto logarithmic subsurface concentrations leads to unrealistic values. These unrealistic concentrations then influence also the surface through the model dynamics. However, unrealistic concentrations can even happen directly at the surface as the following example shows.

To get more insight into the development of the unrealistic concentrations, we examine the profiles of chlorophyll concentration at different dates at two locations where extremely high concentrations are visible in Fig. 9: in the Gulf of Bothnia at $19.79^{\circ} \mathrm{E}, 62.73^{\circ} \mathrm{N}$ and in the Gulf of Finland at $27.54^{\circ} \mathrm{E}, 60.33^{\circ} \mathrm{N}$ (see Fig. 1 for the locations). The left panel of Fig. 10 shows the chlorophyll concentration in the Gulf of Bothnia. The profile looks still realistic on April 22nd. However, a deep maximum develops from April 23rd around $40 \mathrm{~m}$ depth. This maximum continues to grow to extreme values and, due to the model dynamics, also leads to an unrealistic concentration increase towards the ocean surface. The chlorophyll concentration is computed from the concentration of the three phytoplankton groups of ERGOM. Of these, the diatoms and the flagellates show unrealistically high subsurface concentrations, while the concentration of cyanobacteria remains realistic. The largest increases to the concentrations at this location happen during the analysis step. This behaviour shows that in the course of the assimilation process, large cross-covariances developed between the SST and the sub-surface concentrations of diatoms and flagellates, which lead to unrealistic assimilation updates in the linear regression.

The right panel of Figure 10 shows the development of the chlorophyll concentration profile in the Gulf 
of Finland. Here, the Baltic Sea is rather shallow and the profile is initially homogeneous, even though with rather high concentrations of about $40 \mathrm{mg} / \mathrm{m}^{3}$. However, on April 28th the profile becomes more variable with a maximum concentration at the surface and a minimum at around $16 \mathrm{~m}$ depth. Afterwards, the profile jumps to unrealistically high concentrations with a strong gradient from below $13 \mathrm{~m}$ and very low chlorophyll at the bottom. This gradient becomes even steeper in the following analysis steps. The high concentrations of chlorophyll are caused by high concentrations of flagellates, while the concentrations of diatoms and cyanobacteria remain low. The temperature increments by the data assimilation between April 20th and 30th in the eastern Gulf of Finland are always negative. The step-wise increase of the flagellates (and hence chlorophyll) concentration shows that the concentration is negatively correlated with the temperature during this time period. Given the larger assimilation effect with logarithmic concentrations, the unrealistically high concentrations develop. Actually, this effect is, to a lower extent, also visible in the experiment STRONG-lin with actual concentrations when all fields of the BGC model are updated by the data assimilation. In STRONG-lin, the concentrations increase to 170 $\mathrm{mg} / \mathrm{m}^{3}$ in the eastern Gulf of Finland until May 15th (the top right panel of Fig. 9 shows increased concentrations already on May 1st). So also in this case the concentrations are not fully realistic. However, they are much lower than the concentrations obtained for STRONG-log and relax to realistic concentration levels until end of May. Overall, the assimilation in the experiment STRONG-lin behaves stable, while in the case of STRONG-log the concentrations grow to extreme values and don't recover from this. However, if the phytoplankton variables are excluded from the assimilation update of STRONG-lin, their concentrations, including those of the chlorophyll, remain realistic. Thus, the cross-covariances between SST and the phytoplankton fields are not sufficiently well estimated to generate a realistic assimilation update at all times. This might be due to the larger errors in the BGC model state so that the linear regression between the SST and the concentrations fails. 


\section{Conclusion}

In this study, the effect of assimilating satellite sea surface temperature (SST) data into a coupled ocean-biogeochemical model for the North and Baltic Seas has been studied. The model uses nested model grids to better represent the circulation in the German coastal areas. The assimilation is successful in constraining physical ocean fields, which has been assessed with in independent situ data for surface temperature and salinity. With regard to the biogeochemical (BGC) fields, both weakly and strongly-coupled data assimilation have been assessed. With weakly-coupled assimilation, the assimilation only directly updates the physical variables while the BGC fields react dynamically on the changed physical conditions during the following forecast phase. In this case, most BGC model fields are only slightly changed, e.g. oxygen by up to $5 \%$. The changes are particularly small in the North Sea. In the Baltic Sea, the phytoplankton concentrations and the chlorophyll and oxygen are slightly increased as a response to the assimilation. The validation with in situ data did only show small changes in the BGC fields. However, over the full experiment from April to June 2012 the improvements of oxygen concentrations were statistically significant.

In case of strongly-coupled assimilation, both the physical and BGC model fields are directly updated by the data assimilation method. When the actual concentrations of the BGC fields are used in the state vector, the assimilation behaves stable. The changes to the BGC fields are, as expected, larger than for the weakly-coupled assimilation. Quite high concentrations of phytoplankton and hence also chlorophyll appeared in the eastern Gulf of Finland between end of April and middle of May if all BGC fields are updated by the assimilation. These high concentrations disappeared until the end of May and the assimilation was overall stable. In contrast, the concentrations remained realistic if the phytoplankton variables are excluded from the assimilation update, so that only the nutrients and oxygen are directly updated. Thus, only updating the nutrients and oxygen when assimilating SST data appears to be the recommended approach.

The strongly-coupled assimilation was also performed using the logarithm of the BGC field concentrations, which is the common choice when satellite chlorophyll observations are assimilated. In this case, the assimilation 
becomes unstable and local patches of unrealistically high or low concentrations developed. This was mainly the case in the Baltic Sea, but also in the Norwegian Trench. The development of the chlorophyll was examined at two locations in the Baltic Sea, where particularly high concentrations developed. Vertical profiles showed that in the Gulf of Bothnia, the assimilation resulted in an unrealistic sub-surface maximum of chlorophyll around 40 $\mathrm{m}$ depth, caused by high concentrations of diatoms and flagellates. Ultimately this maximum also influenced the concentrations at the surface. In the shallow eastern Gulf of Finland, the assimilation increased the concentrations of flagellates and hence chlorophyll over most of the upper part of the water column. When a vertical localisation was introduced, so that the assimilation increments are linearly reduced as a function of depth until they are set to zero below a specified depth, the assimilation was stabilised in the North Sea. However, in the Baltic unrealistically high concentrations even appeared with a vertical localisation that only changed the upper 5 meters ( 3 model layers).

The results from the weakly-coupled assimilation show that in the North and Baltic Seas the assimilation of only SST data can improve the oxygen concentrations. This improvement is even larger for strongly-coupled assimilation, because of the correlation between temperature and oxygen concentrations. The effect on other BGC model fields was small, but there was no obvious deterioration. This is in contrast to other studies that performed physical data assimilation in the North Atlantic (Berline et al., 2007) or the California Current System (Raghukumar et al., 2015). The application of strongly-coupled assimilation with actual BGC concentrations showed that the cross-covariances between the SST and the BGC fields only lead to changes that were small compared to the errors in the BGC fields. The limited in situ data was not sufficient to provide a clear result whether the changes to the BGC fields are significant.

The differences in the strongly-coupled assimilation using actual concentrations compared to logarithmic concentrations showed a clear advantage of actual concentrations. The assimilation using actual concentrations lead to a more stable assimilation process and more realistic model fields while with logarithmic concentrations unrealistic values were obtained. The application of a vertical localisation lead to a clear improvement, but did 
not solve the issue of unrealistic concentrations in the Baltic Sea. Further, updating only nutrients and oxygen improved the results. To this end, the experiments indicate that for strongly-coupled assimilation between model physics and BGC model variables, the actual concentrations should be used.

\section{Acknowledgement}

This work was carried out within the project MeRamo by the German Federal Ministry of Transportation and Digital Infrastructure (BMVI) through the German Aerospace Center (DLR). We thank the German Oceanographic Data Center and International Council for the Exploration of the Sea (ICES Dataset on Ocean Hydrography. The International Council for the Exploration of the Sea, Copenhagen. 2016) for providing the in situ data. 


\section{References}

Anderson JL (2003) A local least squares framework for ensemble filtering. Mon Wea Rev 131:634-642

Anderson LA, Robinson AR, Lozano CJ (2000) Physical and biological modeling in the Gulf Stream region: I. Data assimilation methodology. Deep Sea Res I 47:1787-1827

Barth A, Alvera-Azcarate A, Beckers JM, Rixen M, Vandenbulcke L (2007) Multigrid state vector for data assimilation in a two-way nested model of the Ligurian Sea. J Mar Syst 65:41-59

Barth A, Alvera-Azcarate A, Gurgel KW, Staneva J, Port A, Beckers JM, Stanev EV (2010) Ensemble perturbation smoother for optimizing tidal boundary conditions by assimilation of high-frequency radar surface currents application to the German Bight. Ocean Sci 6:161-178

Berline L, Brankart JM, Brasseur P, Ourmier'es Y, Verron J (2007) Improving the physics of a coupled physicalbiogeochemical model of the North Atlantic through data assimilation: Impact on the ecosystem. J Mar Syst 64:153-172

Bruening T, Janssen F, Kleine E, Komo H, Massmann S, Menzenhauer-Schuhmacher I, Jandt S, Dick S (2014) Operational ocean forecasting for German coastal waters. Die Küste 81:273-290

Burgers G, van Leeuwen PJ, Evensen G (1998) On the analysis scheme in the ensemble Kalman filter. Mon Wea Rev 126:1719-1724

Campbell JW (1995) The lognormal distribution as a model for bio-optical variability in the sea. J Geophys Res 100(C7):13237-13254

Carmillet V, Brankart JM, Brasseur P, Drange H, Evensen G, Verron J (2001) A singular evolutive extended Kalman filter to assimilate ocean color data in a coupled physical-biochemical model of the North Atlantic ocean. Ocean Modeling 3:167-192 
Ciavatta S, Torres R, Suax-Picart S, Allen JI (2011) Can ocean color assimilation improve biogeochemical hindcasts in shelf seas? J Geophys Res 116:C12043

Ciavatta S, Brewin RJW, Skakala J, Polimene L, de Mora L, Artioli Y, Allen JI (2018) Assimilation of ocean-color plankton functional types to improve marine ecosystem simulations. J Geophys Res Oceans 123:834-654

Dick S, Kleine E, Müller-Navarra SH, Klein SH, Komo H (2001) The operational circulation model of BSH (BSHcmod): model description and validation. Tech. rep., Bundesamt für Seeschiffahrt und Hydrographie, Hamburg, Germany

Doron M, Brasseur P, Brankart JM (2011) Stochastic estimation of biogeochemical parameters of a 3D ocean couples physical-biogeochemical model: Twin experiments. J Mar Syst 87:194-207

Doron M, Brasseur P, Brankart JM, Losa SN, Melet A (2013) Stochastic estimation of biogeochemical parameters from Globcolour ocean color satellite data in a North Atlantic 3D ocean coupled physical-biogeochemical model. J Mar Syst 117:81-95

Evensen G (1994) Sequential data assimilation with a nonlinear quasi-geostrophic model using Monte Carlo methods to forecast error statistics. J Geophys Res 99(C5):10143-10162

Ford D, Barciela R (2017) Global marine biogeochemical reanalyses assimilating two different sets of merged ocean colour products. Rem Sens Env 203:40-54

Ford DA, Edwards KP, Lea D, Barciela RM, Martin MJ, Demaria J (2012) Assimilating GlobColour ocean colour data into a pre-operational physical-biogeochemical model. Ocean Sci 8:751-771

Gaspari G, Cohn SE (1999) Construction of correlation functions in two and three dimensions. Q J Roy Meteor Soc $125: 723-757$

Gregg WW (2008) Assimilation of SeaWiFS ocean chlorophyll data into a three-dimensional global ocean model. J Mar Syst 69:205-225 
Hibler WD (1979) A dynamics/thermodynamic sea ice model. J Phys Oce 9:815-846

Hu C, Lee Z, Franz B (2012) Chlorophyll a algorithms for oligotrophic oceans: A novel approach based on threeband reflectance difference. J Geophys Res Oceans 117(C01011)

Janssen F, Schrum C, Backhaus JO (1999) A climatological data set of temperature and salinity for the Baltic Sea and the North Sea. Deutsche Hydrographische Zeitschrift 51(Suppl 9):5-245

Kleine E (2003) A class of hybrid vertical co-ordinates for ocean circulation modelling. In: Proceedings of the 6th HIROMB scientific workshop, Morzaschita, St. Petersburg, pp 7-15

Liu Y, Fu W (2018) Assimilating high-resolution sea surface temperature data improves the ocean forecast potential in the Baltic Sea. Ocean Sci 14:525-541

Lorkowski I, Paetsch J, Moll A, Kuehn W (2012) Interannual variability of carbon fluxes in the North Sea from 1970 to 2006 - competing effects of abiotic and biotic drivers on the gas-exchange of CO2. Estuarine, Coastal and Shelf Science 100:38-57

Losa SN, Danilov S, Schröter J, Nerger L, Massmann S, Janssen F (2012) Assimilating NOAA SST data into the BSH operational circulation model for the North and Baltic Seas: Inference about the data. J Mar Syst 105-108:152-162

Losa SN, Danilov S, Schröter J, Janjić T, Nerger L, Janssen F (2014) Assimilating NOAA SST data into BSH operational circulation model for the North and Baltic Seas: Part 2. Sensitivity of the forecast's skill to the prior model error statistics. J Mar Syst 129:259-270

Maar M, Moller EF, Larsen J, Madsen KS, Wan Z, She J, Jonasson L, Neumann T (2011) Ecosystem modelling across a salinity gradient from the North Sea to the Baltic Sea. Ecological Modelling 222:1696-1711

Mattern JP, Dowd M, Fennel K (2010) Sequential data assimilation applied to a physical-biological model for the Bermuda Atlantic time series station. J Mar Syst 79:144-156 
Mattern JP, Song H, Edwards CA, Moore AM, Fiechter J (2017) Data assimilation of physical and chlorophyll a observations in the California Current System using two biogeochemical models. Oce Mod 109:55-71

Natvik LJ, Evensen G (2003) Assimilation of ocean colour data into a biochemical model of the North Atlantic. Part 1. Data assimilation experiments. J Mar Syst 40-41:127-153

Nerger L, Gregg WW (2007) Assimilation of SeaWiFS data into a global ocean-biogeochemical model using a local SEIK filter. J Mar Syst 68:237-254

Nerger L, Gregg WW (2008) Improving assimilation of SeaWiFS data by the application of bias correction with a local SEIK filter. J Mar Syst 73:87-102

Nerger L, Hiller W (2013) Software for ensemble-based data assimilation systems - implementation strategies and scalability. Computers \& Geosciences 55:110-118

Nerger L, Hiller W, Schröter J (2005) A comparison of error subspace Kalman filters. Tellus 57A:715-735

Nerger L, Danilov S, Hiller W, Schröter J (2006) Using sea level data to constrain a finite-element primitiveequation ocean model with a local SEIK filter. Ocean Dynamics 56:634-649

Nerger L, Janjić T, Schröter J, Hiller W (2012a) A regulated localization scheme for ensemble-based Kalman filters. Q J Roy Meteor Soc 138:802-812

Nerger L, Janjić T, Schröter J, Hiller W (2012b) A unification of ensemble square root Kalman filters. Mon Wea Rev 140:2335-2345

Nerger L, Losa S, Bruening T, Janssen F (2016) The HBM-PDAF assimilation system for operational forecasts in the North and Baltic Seas. In: Buch E, Antoniou Y, Eparkhina D, Nolan G (eds) Operational Oceanography for Sustainable Blue Growth. Proceedings of the Seventh EuroGOOS International Conference. 28-30 October 2014, Lisbon, Portugal

Neumann T (2000) Towards a 3D-ecosystem model in the Baltic Sea. J Mar Syst 25:405-419 
Neumann T, Siegel H, Gerth M (2015) A new radiation model for Baltic Sea ecosystem modelling. J Mar Syst 152:83-91

Ourmières Y, Brasseur P, Lévy M, Brankart JM, Verron J (2009) On the key role of nutrient data to constrain a coupled physical-biogeochemical assimilative model for the North Atlantic Ocean. J Mar Syst 75:100-115

Park JY, Stock CA, Yang X, Dunne JP, Rosati A, John J, Zhang S (2018) Modeling global ocean biogeochemistry with physical data assimilation: A pragmatic solution to the equatorial instability. J Adv Model Earth Syst 10:891-906

Penny SG, Akella S, Alves O, Bishop C, Buehner M, Chevalier M, Counillon F, Drper C, Frolov S, Fujii Y, Kumar A, Laloyaux P, Mahfouf JF, MArtin M, Pena M, de Rosnay P, Subramanian A, Tardif R, Wang Y, Wu X (2017) Coupled data assimilation for integrated earth system analysis and prediction: Goals, challenges and recommendations. Tech. Rep. WWRP 2017-3, World Meteorological Organization

Pham DT, Verron J, Roubaud MC (1998) A singular evolutive extended Kalman filter for data assimilation in oceanography. J Mar Syst 16:323-340

Pradhan HK, Voelker C, Losa SN, Bracher A, Nerger L (2019) Assimilation of global total chlorophyll OC-CCI data and its impact on individual phytoplankton fields. J Geophys Res Oceans 140:470-490

Raghukumar K, Edwards CA, Goebel NL, Broquet G, Veneziani M, Moore AM, Zehr JP (2015) Impact of assimilating physical oceanographic data on modeled ecosystem dynamics in the California Current System. Prog Ocean 138:546-558

Shulman I, Frolov S, Anderson S, Penta B, Gould R, Sakalaukus P, Ladner S (2013) Impact of bio-optical data assimilation on short-term coupled physical, bio-optical model predictions. J Geophys Res Oceans 118:22152230

Song H, Edwards CA, Moore AM, Fiechter J (2016a) Data assimilation in a coupled physical-biogeochemical 
model of the California Current System using an incremental lognormal 4-dimensional variational approach: Part 1 - model formulation and biological data assimilation twin experiments. Oce Mod 106:131-145

Song H, Edwards CA, Moore AM, Fiechter J (2016b) Data assimilation in a coupled physical-biogeochemical model of the California Current System an incremental lognormal 4-dimensional variational approach: Part 2 joint physical and biological data assimilation twin experiments. Oce Mod 106:146-158

Song H, Edwards CA, Moore AM, Fiechter J (2016c) Data assimilation in a coupled physical-biogeochemical model of the California Current System using an incremental lognormal 4-dimensional variational approach: Part 3 - assimilation in a realistic context using satellite and in situ observations. Oce Mod 106:159-172

Teruzzi A, Dobricic S, Solidoro C, Cossarini G (2014) A 3-D variational assimilation scheme in coupled transportbiogeochemical models: Forecast of Mediterranean biogeochemical properties. J Geophys Res Oceans 119:200217

Vetra-Carvalho S, van Leeuwen PJ, Nerger L, Barth A, Altaf MU, Brasseur P, Kirchgessner P, Beckers JM (2018) State-of-the-art stochastic data assimilation methods for high-dimensional non-Gaussian problems. Tellus A $70(1): 1445364$

While J, Haines K, Smith G (2010) A nutrient increment method for reducing bias in global biogeochemical models. J Geophys Res Oceans 115:C10036

Yu L, Fennel K, Bertino L, Gharamti ME, Thompson KR (2018) Insights on multivariate updates of physical and biogeochemical ocean variables using an ensemble Kalman filter and an idealized model of upwelling. Oce Mod $126: 13-28$ 
Table 1: RMS error and bias with regard to in situ data for both model grids for the FREE run and the forecast and analysis from the experiment WEAK for the period April to July 2012. The upper rows show the errors and bias for SST in ${ }^{\circ} \mathrm{C}$, the lower for surface salinity. The second column shows the the number of collocation points.

\begin{tabular}{|c|c|c|c|c|c|c|c|}
\hline \multicolumn{8}{|c|}{ Surface Temperature $\left({ }^{\circ} \mathrm{C}\right)$} \\
\hline & & \multicolumn{3}{|c|}{ RMSE } & \multicolumn{3}{|c|}{ Bias } \\
\hline grid & no. points & free & forecast & analysis & free & forecast & analysis \\
\hline coarse & 6674 & 1.070 & 0.925 & 0.920 & 0.482 & 0.300 & 0.297 \\
\hline fine & 800 & 1.151 & 1.053 & 1.052 & 0.424 & 0.247 & 0.246 \\
\hline \multicolumn{8}{|c|}{ Surface Salinity (psu) } \\
\hline & & \multicolumn{3}{|c|}{ RMSE } & \multicolumn{3}{|c|}{ Bias } \\
\hline grid & no. points & free & forecast & analysis & free & forecast & analysis \\
\hline coarse & 6472 & 1.430 & 1.387 & 1.385 & -0.266 & -0.222 & -0.217 \\
\hline fine & 796 & 2.763 & 2.770 & 2.773 & 0.732 & 0.617 & 0.617 \\
\hline
\end{tabular}

Table 2: RMS error and bias of biogeochemical fields with regard to in situ data at the surface for both model grids and the FREE run and forecast and analysis from the experiment WEAK for the period April to July 2012. Shown is also the number of collocation points. The units are $\mathrm{mmol} \mathrm{N} / \mathrm{m}^{3}$ for ammonium and nitrate, $\mathrm{mmol} \mathrm{P} / \mathrm{m}^{3}$ for phosphate, $\mathrm{mmol} \mathrm{O} / \mathrm{m}^{3}$ for oxygen, $\mathrm{mmol} \mathrm{Si} / \mathrm{m}^{3}$ for silicate, and $\mathrm{mg} \mathrm{Chl} / \mathrm{m}^{3}$ for Chlorophyll.

\begin{tabular}{|c|c|c|c|c|c|c|}
\hline \multicolumn{7}{|c|}{ RMSE } \\
\hline & \multicolumn{3}{|c|}{ Coarse grid } & \multicolumn{3}{|c|}{ Fine grid } \\
\hline field & free & analysis & no. points & free & analysis & no. points \\
\hline Ammonium & 1.562 & 1.561 & 1146 & 1.393 & 1.394 & 228 \\
\hline Nitrate & 11.116 & 10.810 & 1372 & 12.914 & 13.118 & 366 \\
\hline Phosphate & 0.421 & 0.421 & 1392 & 0.303 & 0.299 & 366 \\
\hline Chlorophyll & 8.203 & 8.205 & 1428 & 5.781 & 5.783 & 306 \\
\hline Oxygen & 39.595 & 38.195 & 1494 & 34.297 & 34.800 & 426 \\
\hline Silicate & 17.979 & 18.092 & 1188 & 8.361 & 8.404 & 366 \\
\hline \multicolumn{7}{|c|}{ Bias } \\
\hline & \multicolumn{3}{|c|}{ Coarse grid } & \multicolumn{3}{|c|}{ Fine grid } \\
\hline field & free & analysis & no. points & free & analysis & no. points \\
\hline Ammonium & -0.428 & -0.430 & 1146 & -0.643 & -0.643 & 228 \\
\hline Nitrate & 3.154 & 3.071 & 1372 & 3.760 & 3.622 & 366 \\
\hline Phosphate & 0.035 & 0.033 & 1392 & 0.083 & 0.078 & 366 \\
\hline Chlorophyll & -2.208 & -2.207 & 1428 & -1.34 & -1.325 & 306 \\
\hline Oxygen & -17.030 & -14.192 & 1494 & -3.117 & -1.114 & 426 \\
\hline Silicate & 3.040 & 3.038 & 1188 & -3.343 & -3.404 & 366 \\
\hline
\end{tabular}


Table 3: RMS error and bias of biogeochemical fields of the data assimilation analysis state with regard to in situ data at the surface for both model grids from the experiment STRONG-lin for the period April to July 2012. Shown are the cases that all BGC variables are updated by the data assimilation (columns 'full BGC') and that the phytoplankton variables are excluded from the update ('nutrients only'). The units are the same as in Tab. 2

\begin{tabular}{|c||c|c||c|c||c|c|c|c|}
\hline \multicolumn{1}{|c||}{ Update } & \multicolumn{4}{c||}{ full BGC } & \multicolumn{4}{c|}{ nutrients only } \\
\hline \multicolumn{1}{|c||}{} & \multicolumn{2}{c||c}{ coarse grid } & \multicolumn{2}{c|}{ fine grid } & \multicolumn{2}{c|}{ coarse grid } & \multicolumn{2}{c|}{ fine grid } \\
\hline field & RMSE & bias & RMSE & bias & RMSE & bias & RMSE & bias \\
\hline Ammonium & 1.560 & -0.419 & 1.402 & -0.640 & 1.558 & -0.430 & 1.394 & -0.640 \\
Nitrate & 10.903 & 3.293 & 13.055 & 3.750 & 10.812 & 3.229 & 12.803 & 3.679 \\
Phosphate & 0.428 & 0.041 & 0.319 & 0.101 & 0.423 & 0.030 & 0.319 & 0.099 \\
Chlorophyll & 8.360 & -1.71 & 5.830 & -1.217 & 8.183 & -2.204 & 5.800 & -1.298 \\
Oxygen & 37.731 & -12.911 & 34.964 & -0.336 & 37.510 & -13.704 & 34.820 & -0.367 \\
Silicate & 18.246 & 3.508 & 8.339 & -2.885 & 18.177 & 3.557 & 8.239 & -2.785 \\
\hline
\end{tabular}

Table 4: RMS error of biogeochemical fields with regard to in situ data at the surface for both model grids and the FREE run and forecast and analysis from the experiment STRONG-log with logarithmic concentrations for the period April-May 2012. Shown are separate values for the North Sea and the Baltic Sea. Shown are the experiments in which all fields of the BGC model are updated 'full BGC' and where only nutrients and oxygen are update 'nutrients only'. The columns marked 'full vertical' refer to the assimilation without vertical localization, while 'vloc $=10 \mathrm{~m}$ ' refers a to vertical localization of 10 meters. The units are the same as in Tab. 2. The values in italic font indicate fields with unrealistic patterns.

\begin{tabular}{|c||c||c|c|c|c|}
\hline \multicolumn{1}{|c||}{} & \multicolumn{5}{c|}{ North Sea } \\
\hline field & FREE & $\begin{array}{c}\text { STRONG-log } \\
\text { full BGC } \\
\text { full vertical }\end{array}$ & $\begin{array}{c}\text { STRONG-log } \\
\text { full BGC } \\
\text { vloc=10m }\end{array}$ & $\begin{array}{c}\text { STRONG-log } \\
\text { nutrients only } \\
\text { full vertical }\end{array}$ & $\begin{array}{c}\text { STRONG-log } \\
\text { nutrients only } \\
\text { vloc=10m }\end{array}$ \\
\hline Ammonium & 0.98 & 63.31 & 0.98 & 0.97 & 0.97 \\
Nitrate & 13.35 & 1024.2 & 13.34 & 58.5 & 16.12 \\
Phosphate & 0.43 & 27.52 & 0.43 & 0.43 & 0.43 \\
Chlorophyll & 8.81 & 9.26 & 8.80 & 8.76 & 8.78 \\
Oxygen & 37.548 & 11497.1 & 37.559 & 37.56 & 36.56 \\
Silicate & 11.66 & 12.09 & 12.05 & 46.42 & 15.95 \\
\hline \multicolumn{5}{|c|}{ Baltic Sea } \\
Ammonium & 1.30 & 5890.2 & 1499.1 & 7.99 & 1.29 \\
Nitrate & 12.58 & 6934.2 & 88.3 & 2702.2 & 15.21 \\
Phosphate & 0.251 & 3804.3 & 646.7 & 0.25 & 0.26 \\
Chlorophyll & 10.54 & 621.55 & 10.56 & 10.57 & 10.57 \\
Oxygen & 21.785 & 52183.8 & 21.166 & 23.01 & 21.13 \\
Silicate & 15.22 & 1833.8 & 15.18 & 17.06 & 15.18 \\
\hline
\end{tabular}




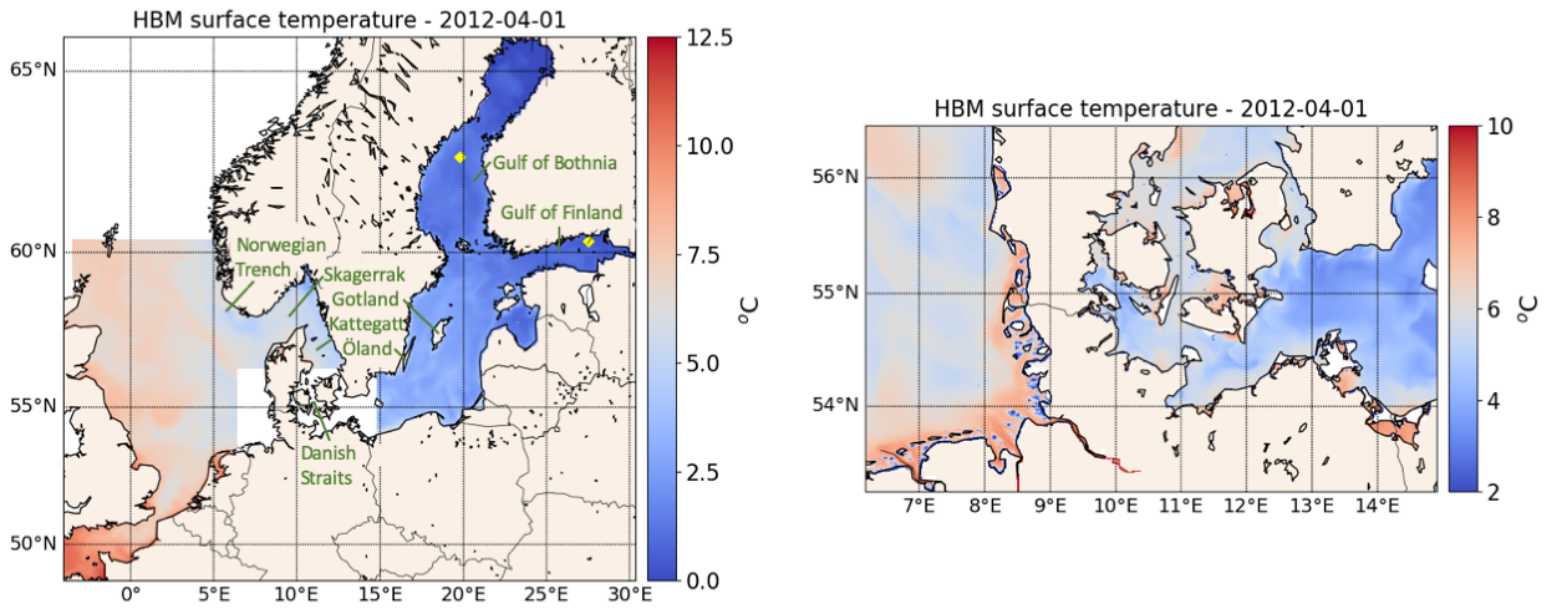

Figure 1: Sea surface temperature on April 1, 2012 on the coarse (left) and fine (right) model domains. The coarse model grid excludes the region of the fine grid. In the left plot some geographic regions discussed in the text are marked. Further, the yellow markers at $19.79^{\circ} \mathrm{E}, 62.725^{\circ} \mathrm{N}$ in the Gulf of Bothnia and at $27.54^{\circ} \mathrm{E}, 60.33^{\circ} \mathrm{N}$ in the Gulf of Finland show the location of profiles that will be discussed in Sec. 7 . 


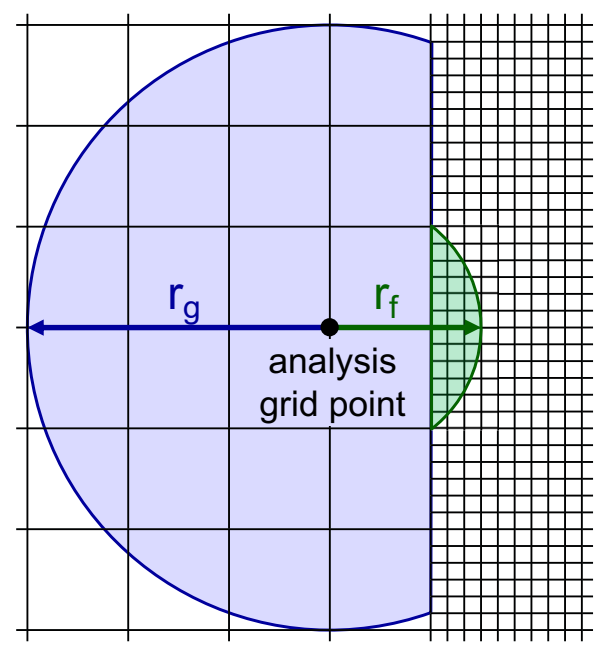

Figure 2: Localisation in nested model grids: The currently updated grid point in the coarse model grid is marked by the black dot. The blue circle marks the radius $r_{\mathrm{g}}$ for which observations on the coarse grid include the analysis grid point. For observations on the fine grid, the corresponding shorter radius $r_{\mathrm{f}}$ is marked by the green circle.
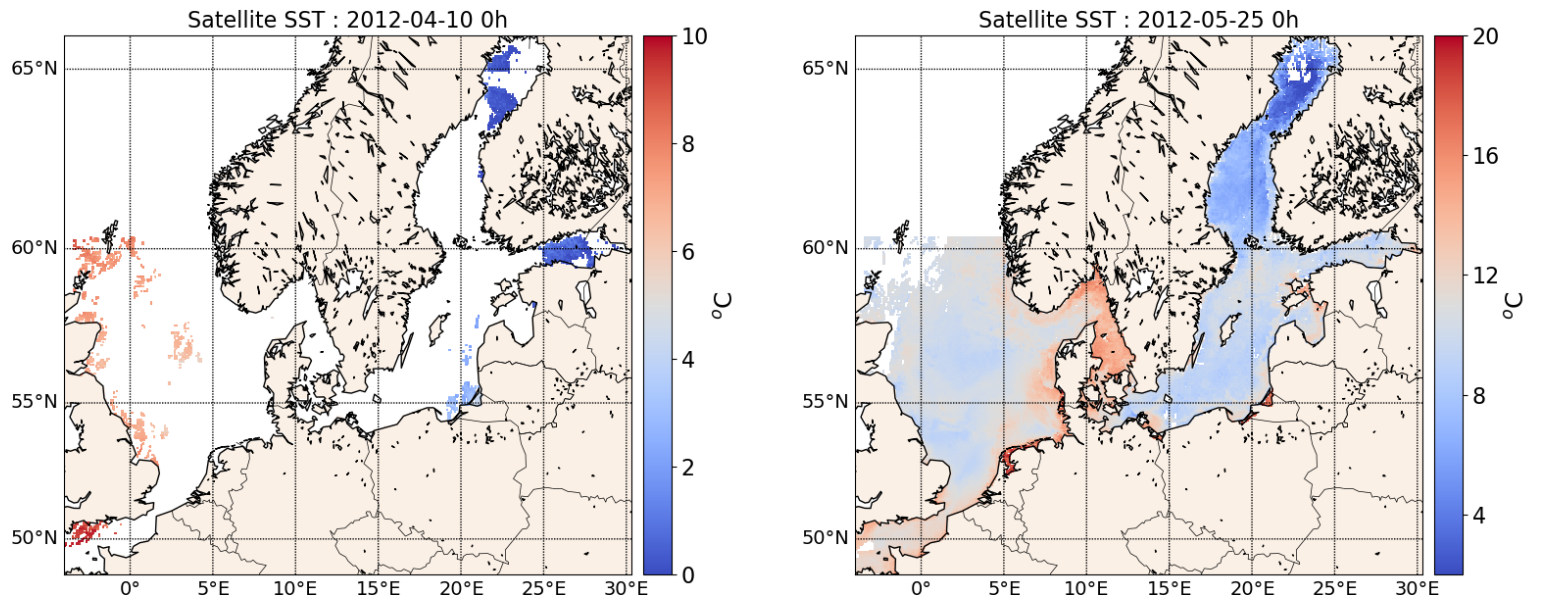

Figure 3: Satellite SST observations on both model grids. Shown are two extremes of data coverage. On April 10, the North and Baltic Seas were nearly fully covered by clouds. On the coarse grid data is only available on $7 \%$ of the grid points, while for the fine mesh there are zero observations over the 12-hour time window. For May 25, the domains were nearly cloud free so that there are only small data-void regions. 

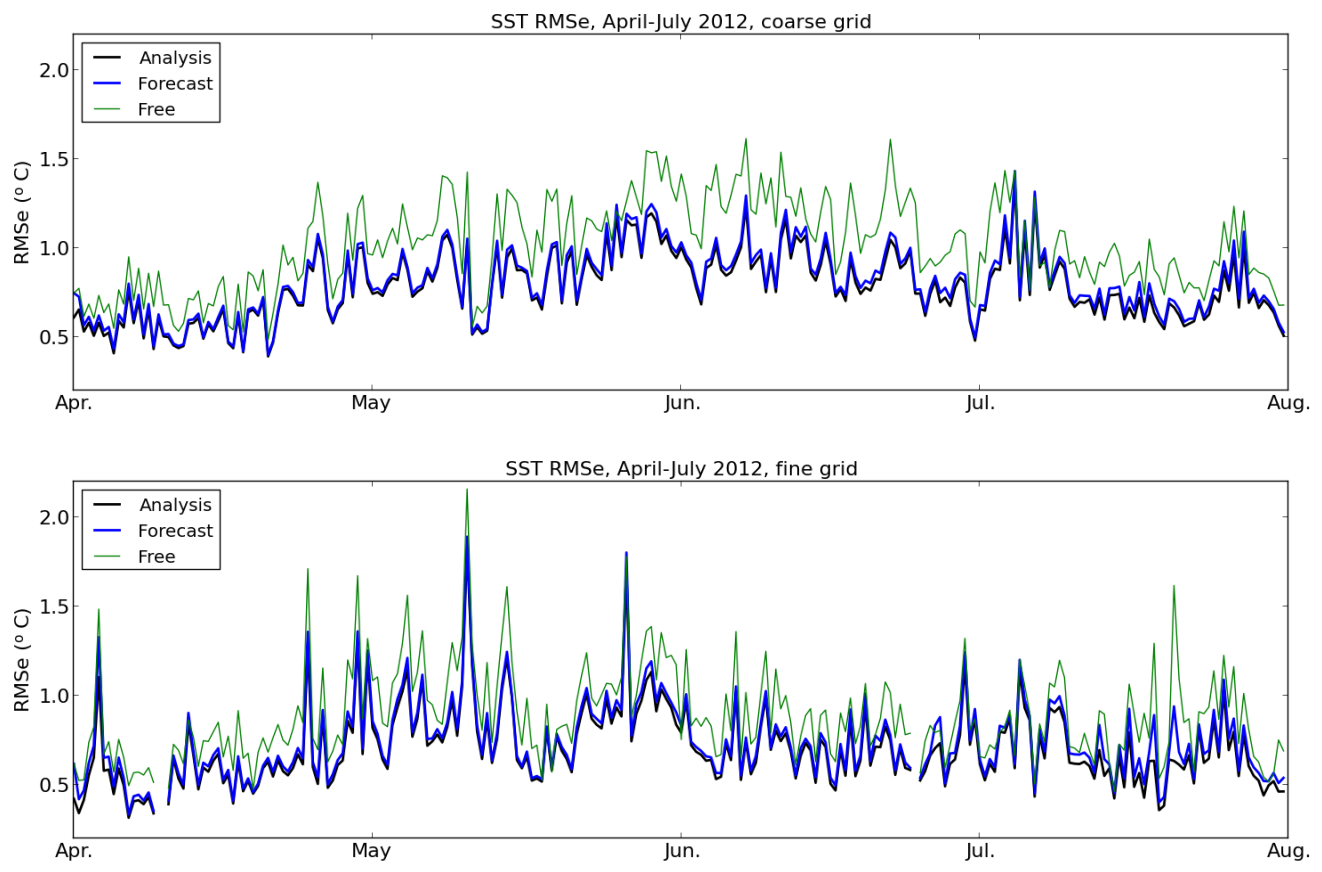

Figure 4: RMS error with regard to the assimilated SST observations over time. The upper panel shows the RMSE for the coarse model grid while the lower panel shows the fine grid. The lines are (green) the RMSE for the free model run, (black) the values directly after the analysis step, and (blue) the RMSE for the 12-hour forecasts. 

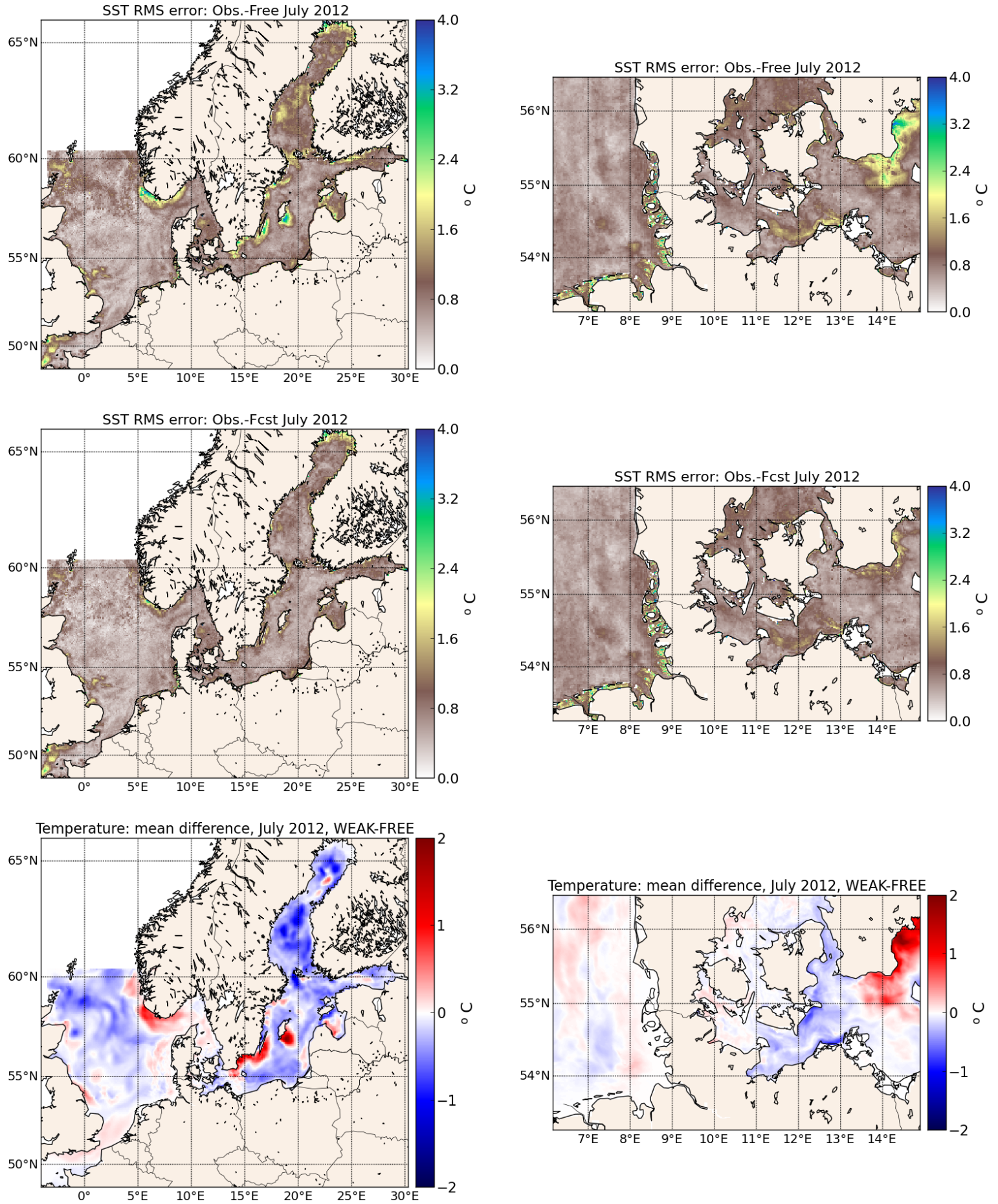

Figure 5: Surface temperature averaged for July 2012. Shown are: (upper row) RMS error with regard to the assimilated observations for the experiment FREE, (middle) RMSE for the experiment WEAK, (bottom) change in temperature due to the assimilation. The assimilation result to changes up to $2^{\circ} \mathrm{C}$ which strongly reduces the RMSE in both grids. 

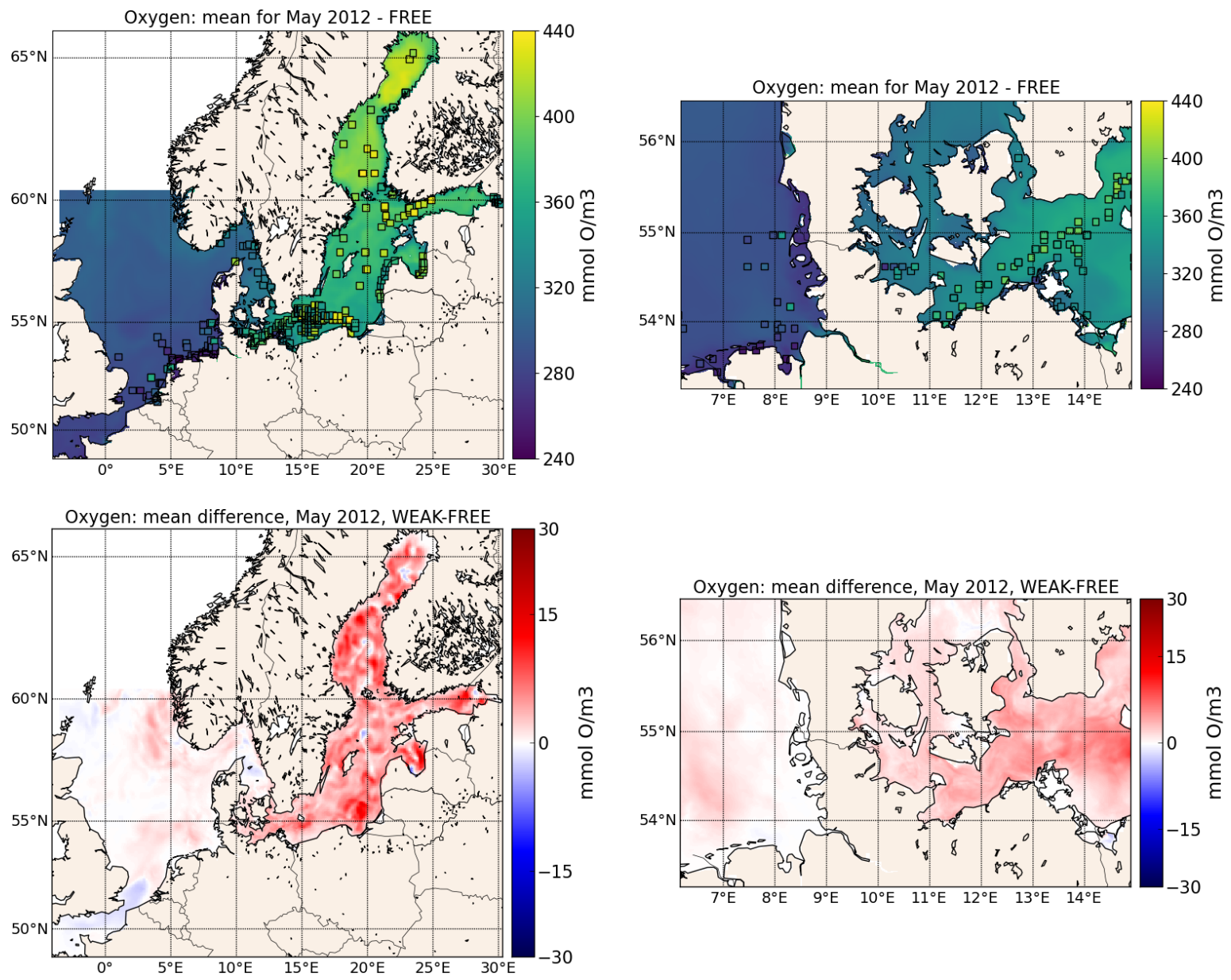

Figure 6: Oxygen at the ocean surface averaged over May 2012 on both model grids. The upper row shows the experiment FREE. Superposed to the model field are the in situ observations displayed as squares. The bottom row shows the mean difference of the experiments WEAK-FREE, i.e. the change in oxygen caused by the data assimilation. The model underestimates the oxygen in particular in the Baltic Sea where the assimilation increases the concentrations. 

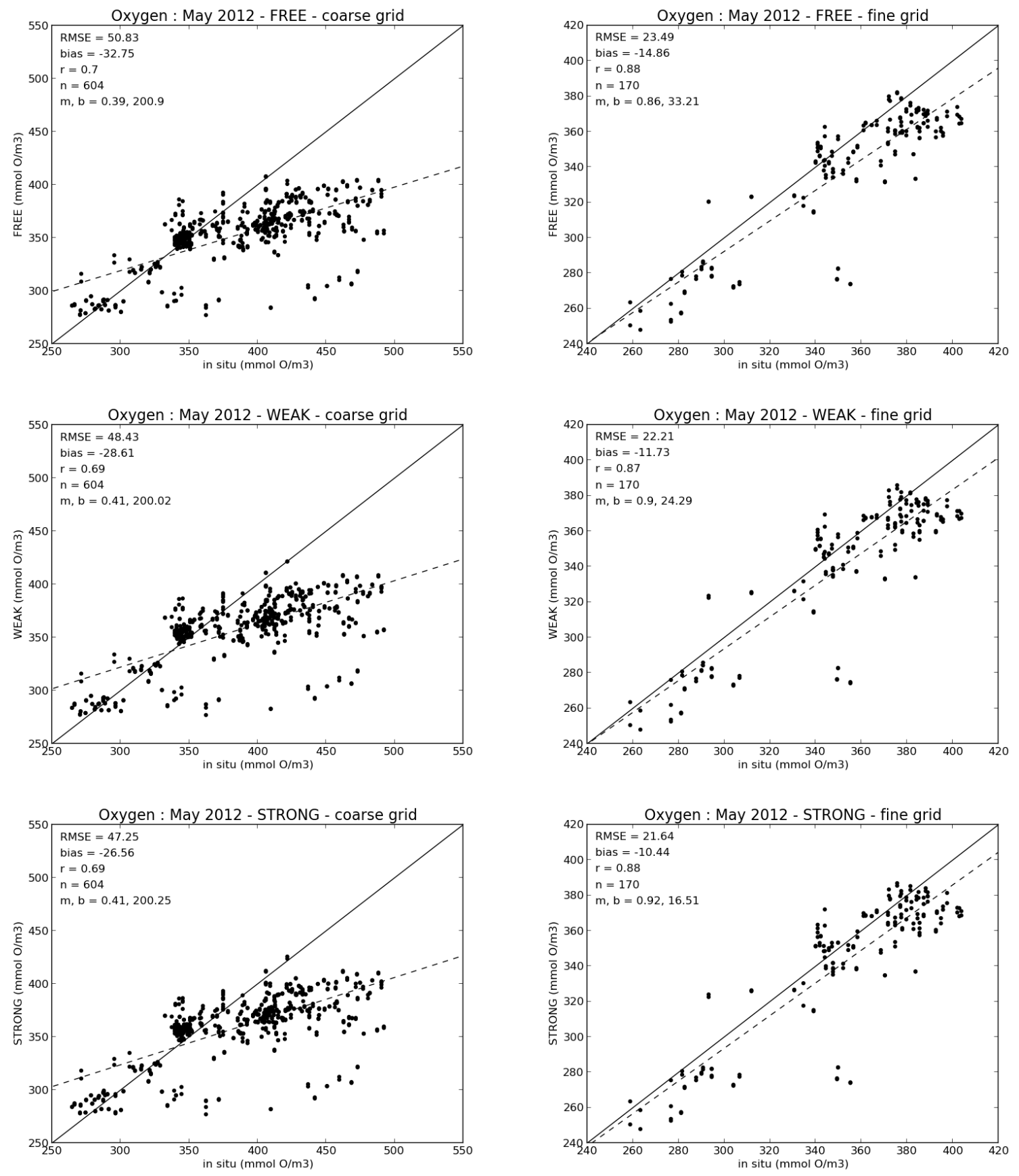

Figure 7: Comparison of the different model simulations with in situ data: experiments FREE (top), WEAK (middle), and STRONG-lin (bottom). The values for the coarse grid are shown in the left column and those for the fine mesh in the right column. 


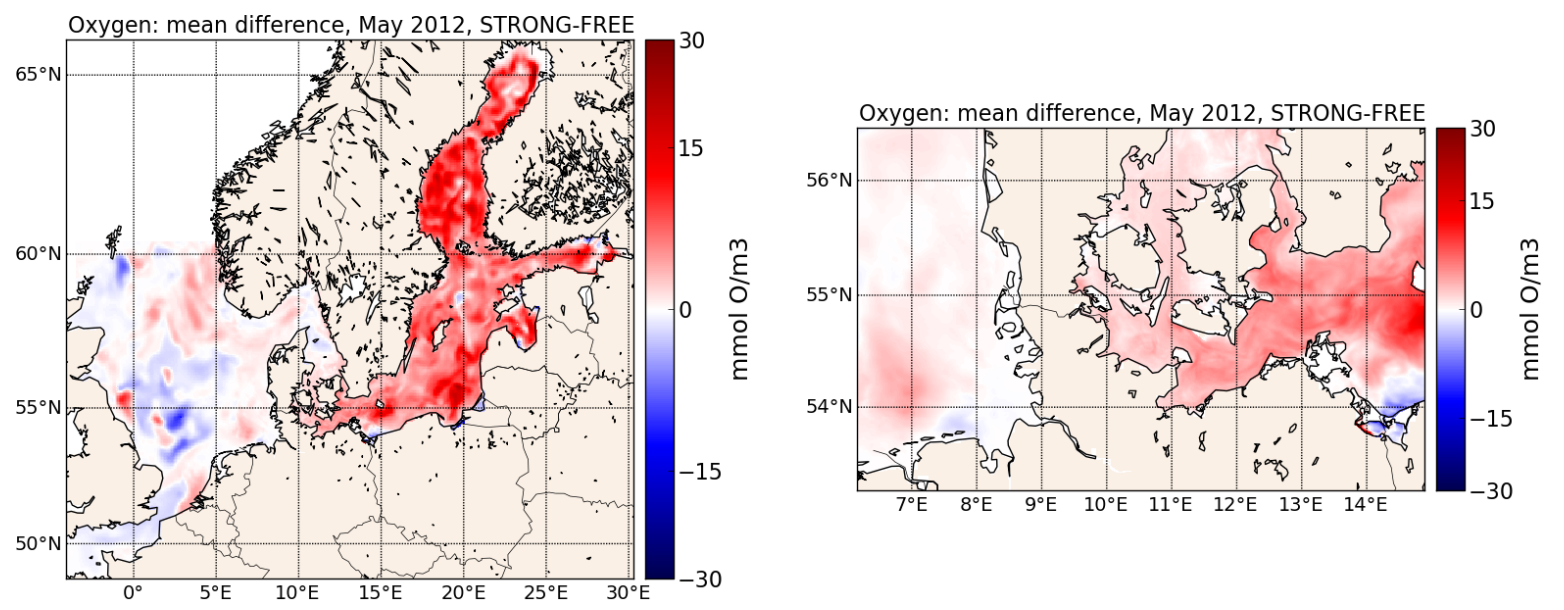

Figure 8: Change in oxygen concentrations caused by the strongly-coupled assimilation experiment STRONG-lin shown as the difference of the experiments STRONG-lin minus FREE. The strongly-coupled assimilation leads to larger changes compared to weakly-coupled assimilation 

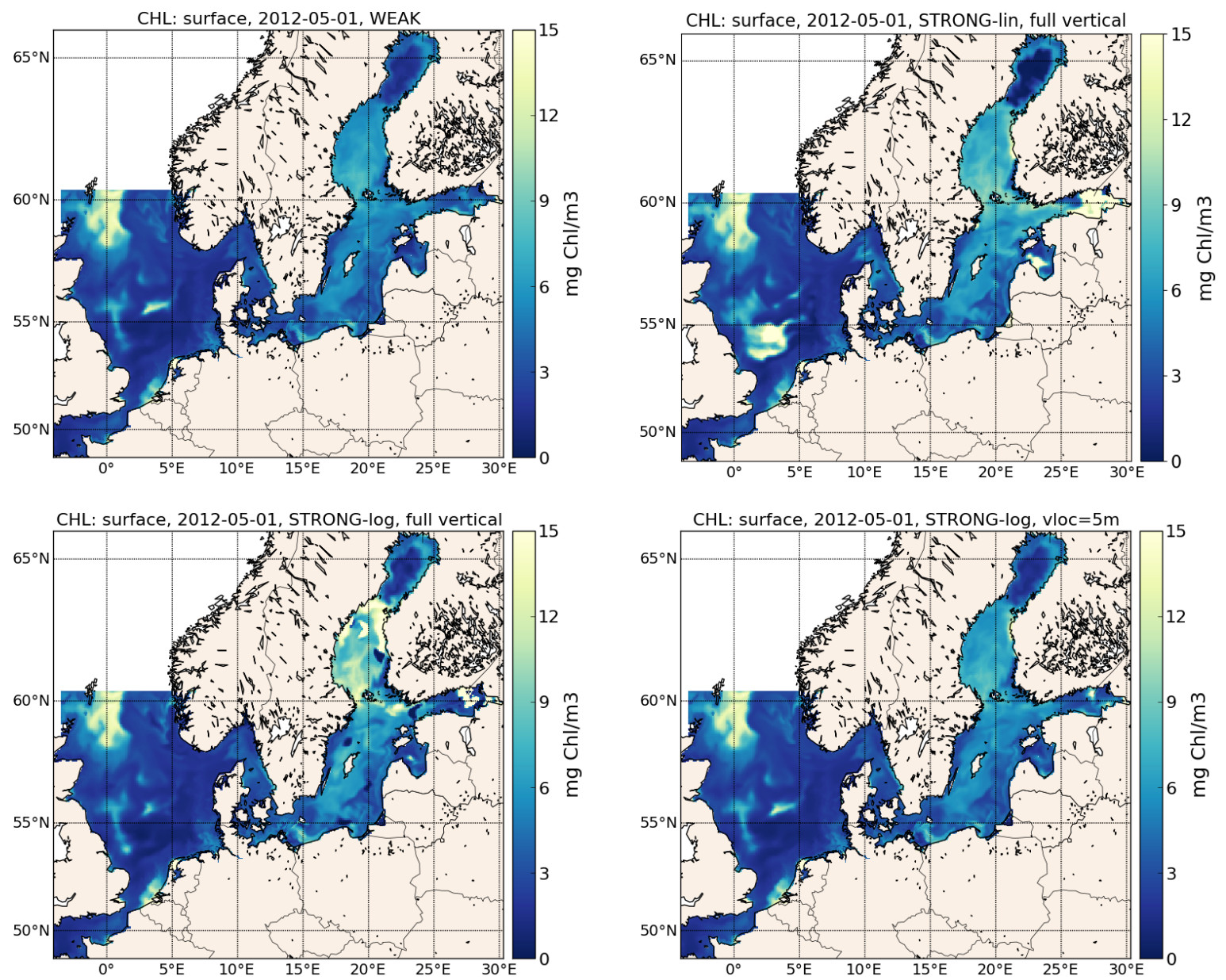

Figure 9: Chlorophyll concentration on May 1, 2012 from experiment (top left) WEAK, (top right) STRONG-lin without vertical localisation, (bottom left) STRONG-log without vertical localisation, and (bottom right) STRONG-log with vertical localisation of $5 \mathrm{~m}$. While the vertical localisation improves the field, there remains an unrealistic high-concentration spot in the eastern Gulf of Finland. 

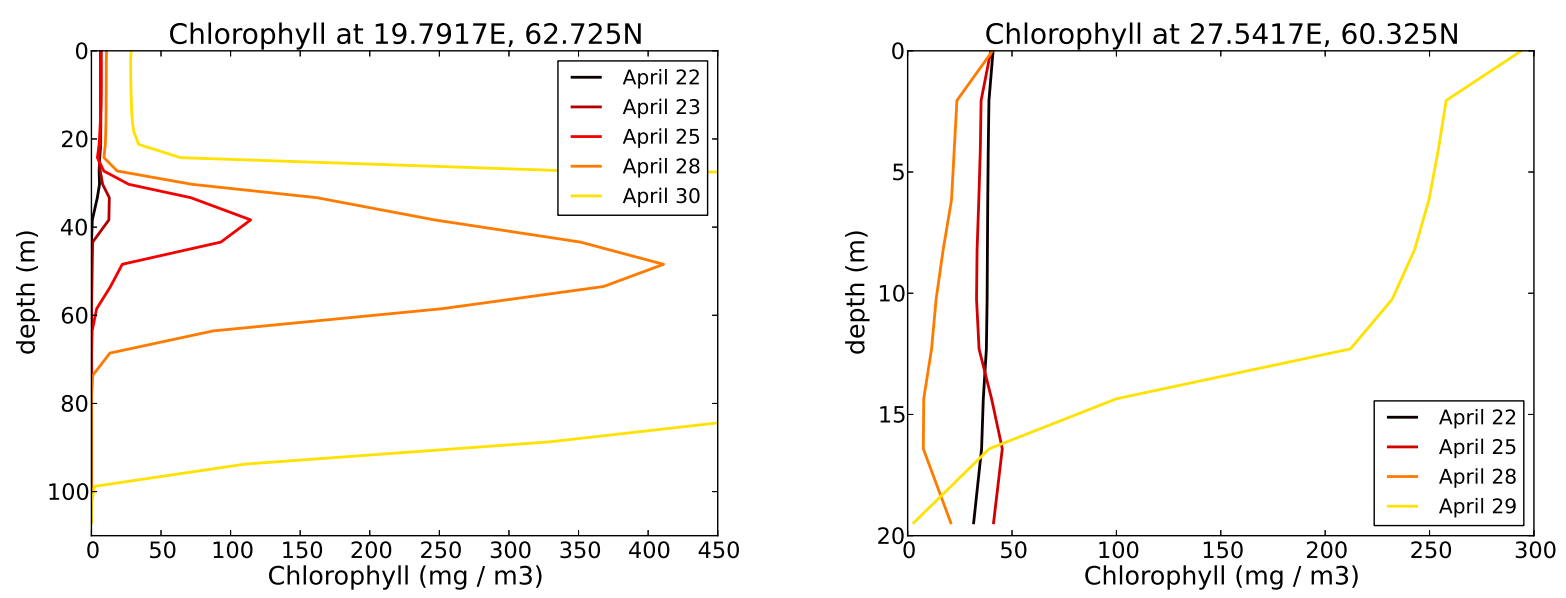

Figure 10: Chlorophyll profiles at four dates in April at two locations where unrealistic concentrations develop: (left) in the Gulf of Bothnia, where first an unrealistic deep maximum develops, (right) In the Gulf of Finland, where the concentration increases over most of the water column. 